\title{
Patterns in the Multiwavelength Behavior of Candidate Neutrino Blazars
}

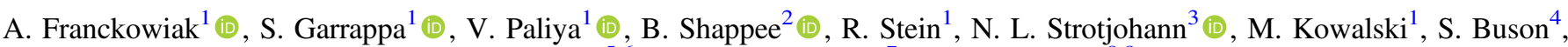 \\ S. Kiehlmann ${ }^{5,6}$, W. Max-Moerbeck ${ }^{7}$, and R. Angioni ${ }^{8,9}$ \\ ${ }^{1}$ Deutsches Elektronen-Synchrotron DESY, D-15738 Zeuthen, Germany; anna.franckowiak@desy.de, simone.garrappa@desy.de, vaidehi.s.paliya@gmail.com \\ ${ }^{2}$ Institute for Astronomy, University of Hawaii, Honolulu, HI 96822, USA \\ ${ }^{3}$ Department of Particle Physics and Astrophysics, Weizmann Institute of Science, Rehovot, 76100, Israel \\ ${ }^{4}$ University of Würzburg, D-97074 Würzburg, Germany \\ ${ }^{5}$ Institute of Astrophysics, Foundation for Research and Technology-Hellas, GR-71110 Heraklion, Greece \\ ${ }^{6}$ Department of Physics, University of Crete, GR-70013 Heraklion, Greece \\ ${ }^{7}$ Departamento de Astronomía, Universidad de Chile, Camino El Observatorio 1515, Las Condes, Santiago, Chile \\ ${ }^{8}$ ASI Space Science Data Center, Via del politecnico, snc. I-00133, Rome, Italy \\ 9 INFN Roma Tor Vergata, Via della Ricerca Scientifica, 1. I-00133, Rome, Italy \\ Received 2020 January 27; revised 2020 March 21; accepted 2020 March 23; published 2020 April 28
}

\begin{abstract}
Motivated by the identification of the blazar TXS $0506+056$ as the first promising high-energy neutrino counterpart candidate, we search for additional neutrino blazar candidates among the Fermi-Large Area Telescope detected blazars. We investigate the multiwavelength behavior from radio to $\mathrm{GeV}$ gamma-rays of blazars found to be in spatial coincidence with single high-energy neutrinos and lower-energy neutrino flare candidates. In addition, we compare the average gamma-ray emission of the potential neutrino-emitting sources to the entire sample of gamma-ray blazars. We find that neutrino-emitting blazar candidates are statistically compatible with hypotheses of both a linear correlation and no correlation between neutrino and gamma-ray energy flux.
\end{abstract}

Unified Astronomy Thesaurus concepts: High energy astrophysics (739); Neutrino astronomy (1100); Blazars (164); Light curves (918)

\section{Introduction}

After the detection of a diffuse flux of high-energy neutrinos (Aartsen et al. 2013), the most pressing challenge is to identify where these neutrinos are produced. Among the prime candidates are active galactic nuclei (AGNs), especially those with a relativistic jet pointing toward us, the so-called blazars (e.g., Stecker et al. 1991; Mannheim et al. 1992; Mannheim 1993, 1995; Szabo \& Protheroe 1994; Mastichiadis 1996; Protheroe 1999; Atoyan \& Dermer 2001; Dimitrakoudis et al. 2012; Murase 2017). No significant clusters of neutrinos in either space or time have been identified by all-sky searches of IceCube data (Aartsen et al. 2015, 2017a, 2020). Searching for neutrinos from a predefined list of 110 sources revealed a $2.9 \sigma$ excess at the position of the Seyfert 2 galaxy NGC 1068 (Aartsen et al. 2020). Combining neutrino with multiwavelength data is the key to probing neutrino emission from various source populations and identifying potential electromagnetic counterparts.

High-energy neutrinos are solely produced in the interaction of cosmic-ray nuclei with ambient matter or photon fields. In either case, both charged and neutral pions are produced. The neutral pions decay into two gamma-rays, and the charged pions produce neutrinos in their decay chain. While gammarays can also be produced in leptonic processes such as synchrotron emission, bremsstrahlung, and inverse Compton scattering, neutrinos are exclusively produced in hadronic processes. They are therefore considered the smoking-gun signature for the identification of cosmic-ray accelerators. Gamma-rays produced alongside high-energy neutrinos can cascade down to lower energies through interactions within the source or during propagation. Increased neutrino activity might therefore be accompanied by increased electromagnetic emission that could appear in various wavelength bands.

The first likely extragalactic neutrino counterpart is the gamma-ray blazar TXS $0506+056$, which was found to be in a flaring state in spatial and temporal coincidence with the arrival of the $290 \mathrm{TeV}$ neutrino event IC-190722A (Aartsen et al. 2018a) at $3 \sigma$ significance. This finding motivated an archival search for lower $\mathcal{O}(1-10 \mathrm{TeV})$ neutrinos from the sky position of TXS $0506+056$, which resulted in the detection of a 160 day long neutrino flare in 2014/15 with $3.5 \sigma$ significance (Aartsen et al. 2018b). Surprisingly, this archival neutrino flare was not accompanied by increased activity in gamma-ray, optical, or radio wavelengths (Aartsen et al. 2018b). Note that no dedicated follow-up campaign was performed at the time of the neutrino flare, and most of the available multiwavelength data were collected by survey instruments. Hints for a hardening of the gamma-ray spectrum during the archival neutrino flare were identified by Padovani et al. (2018) but were not found to be statistically significant $(\leqslant 2 \sigma)$ by Garrappa et al. (2019).

These two neutrino observations from the same source are difficult to reconcile through a single emission model. That the neutrino luminosity of the archival flare is more than four times larger than the gamma-ray luminosity (Aartsen et al. 2018b) suggests a hidden mechanism of neutrino production, e.g., through the attenuation of hadronic gamma-rays due to cascades initiated by photons from the jet or the broad-line region (BLR; Reimer et al. 2019; Rodrigues et al. 2019). We note that Petropoulou et al. (2020), Rodrigues et al. (2019), and Reimer et al. (2019) do not find a set of model parameters explaining the large neutrino flux from the archival neutrino flare without overshooting the electromagnetic observations. Furthermore, those hidden source scenarios (Murase et al. 2016) are inconsistent with the association of IC-170922A with a strong gamma-ray flare from TXS 0506+056 (e.g., Ansoldi et al. 2018; Cerruti et al. 2019; Gao et al. 2019). There have been, however, attempts to explain both observations in a single model by Zhang et al. (2020) and Liu et al. (2019), 
which come at the cost of assuming more complex geometries. The first one assumes a neutral beam scenario, while the second relies on hadronuclear interactions between protons in the jet and material in a dense gas cloud in the vicinity of the black hole.

Accordingly, establishing and understanding either of the scenarios is of significant importance.

The detection of the archival neutrino flare from the direction of TXS 0506+056 motivated a follow-up analysis (O'Sullivan $\&$ Finley 2019) searching for similar neutrino flares from the position of all sources in the third catalog of AGNs detected with the Large Area Telescope (LAT) on board the Fermi Gamma-ray Space Telescope (3LAC; Ackermann et al. 2015). The most significant neutrino flare candidate was derived for each source without accounting for electromagnetic observations of the sources. With neutrino data alone, no significant excess of flares was found above the expected atmospheric background.

A similar situation occurred in the case of TXS 0506+056, where IC-170922A with a signalness of $56 \%$ by itself was not significant, and the archival neutrino flare in 2014/15 was not found significant in an all-sky neutrino-only search. Only with the added information through multiwavelength data was it possible to identify TXS $0506+056$ as the first promising candidate high-energy neutrino source.

The goal of this paper is to better understand blazars as possible source candidates for cosmic neutrinos and their emission mechanisms through the study of their electromagnetic activity. We search for coincidences of single wellreconstructed high-energy $\mathcal{O}(100 \mathrm{TeV})$ neutrino events with blazars detected by the Fermi-LAT. Furthermore, we investigate the multiwavelength behavior for the most significant sources reported by O'Sullivan \& Finley (2019) and sources found spatially consistent with single high-energy neutrinos.

We search for gamma-ray, X-ray, optical, and radio activity correlated with the neutrino emission.

Finally, we study the ensemble of candidate sources by testing for the generic properties expected for neutrino-emitting source populations.

We describe the sample of potential neutrino source candidates in Section 2. Section 3 describes the multiwavelength data used to compile light curves and spectral energy distributions (SEDs) for this study, and Section 4 discusses the statistical methods applied. We present our results in Section 5 and conclude in Section 6.

\section{Source Sample}

The neutrino sample used in this paper comes from the IceCube Neutrino Observatory, a cubic kilometer-scale Cerenkov detector located at the geographic South Pole. A complete description of the IceCube detector is provided in Aartsen et al. (2017b).

\subsection{Neutrino Flare Candidates}

O'Sullivan \& Finley (2019) used a sample of wellreconstructed muon tracks from atmospheric and astrophysical neutrinos in the time period from 2012 April 26 to 2017 May 11. The sample covers the northern sky at declinations above $-5^{\circ}$. The positions of 1023 sources from the 3LAC catalog are searched for a time-dependent neutrino signal in an unbinned maximum-likelihood analysis. The 11 most significant neutrino flares are reported in O'Sullivan \& Finley (2019). Two of the sources, B2 $1126+37$ and MG2 J112910+3702, are the two possible counterparts of 3FGL J1129.0+3705, which corresponds to 4FGL J1129.1+3703 in the fourth catalog of AGNs detected by Fermi-LAT (4LAC; Fermi-LAT collaboration 2019). In 4LAC, the source is associated only with CRATES J112916+370317, which has the same coordinates as MG2 J112910+3702. We therefore only keep CRATES $\mathrm{J} 112916+370317$ in our sample; i.e., we study the remaining 10 sources reported by O'Sullivan \& Finley (2019).

The temporal profile of each neutrino flare candidate is described by a Gaussian, and for each flare, the best-fit central value $T_{0}$ and width $T_{W}$ of the Gaussian are reported. The latter is defined as twice the standard deviation of the Gaussian (see Table 3). O'Sullivan \& Finley (2019) reported pretrial $p$-values for the neutrino flare candidates ranging from $3.3 \times 10^{-3}$ to $3.5 \times 10^{-5}$, but after trial correction, none are significant. They performed a binomial test to assess the statistical significance of the ensemble yielding a $p$-value of $11 \%$, which increases to $24 \%$ if TXS $0506+056$ is removed from the sample, compatible with expectations from the background.

\subsection{Single High-energy Neutrinos}

The IceCube real-time program selects high-energy $(\gtrsim 100 \mathrm{TeV})$ starting and throughgoing muon track events (Aartsen et al. 2017c). A sample of real-time and archival events that would have qualified as a real-time alert but was recorded before the real-time system was operational was searched for blazar-neutrino coincidence (Aartsen et al. 2018a; Garrappa et al. 2019). Both studies focused on wellreconstructed events with a $90 \%$ containment radius of less than $5 \mathrm{deg}^{2}$. In addition to the coincidence of IC-170922A with TXS $0506+056$, the $\sim 100 \mathrm{TeV}$ neutrino IC-141209A was identified in spatial coincidence with the BL Lac object GB6 J1040+0617. A detailed description of the multiwavelength behavior of the two sources can be found in Aartsen et al. (2018a) and Garrappa et al. (2019), respectively. Here we study the IceCube real-time alerts (see Table 1) and archival neutrino events that would have passed the same selection criteria (see Table 2). Between 2016 April and 2019 May, the IceCube collaboration operated two high-energy neutrino alert streams: the extremely high-energy (EHE) stream and the high-energy starting-track stream (HESE). In 2019 June, the alert streams were unified to the GOLD and BRONZE streams, defined by a purity of $50 \%$ and $30 \%$, respectively. Similarly to what was done in Garrappa et al. (2019), we exclude sources with a $90 \%$ angular uncertainty larger than $5 \mathrm{deg}^{2}$ to remove events for which no significant association would be possible given their poor localization. Such events will typically be coincident with many blazars, resulting in a poor association probability.

Between 2016 April and 2019 October, a total of 35 alerts were issued, and 16 survive our selection criteria (see Table 1). Forty archival events have been identified between 2010 September and 2016 May, ${ }^{10}$ and 28 pass our selection (see Table 2). Four additional coincidences are identified. The neutrino event IC-190730A was reported to be in spatial coincidence with the bright gamma-ray blazar PKS $1502+106$ (IceCube Collaboration 2019a). Another coincidence was found with the HESE event IceCube-190221A and two

\footnotetext{
${ }^{10}$ Note that the EHE stream started only in 2016 July, while the HESE stream was operational beginning in 2016 April.
} 
Table 1

Real-time Neutrino Alerts

\begin{tabular}{|c|c|c|c|c|c|c|}
\hline $\begin{array}{l}\text { IceCube } \\
\text { Alert Name }\end{array}$ & Signalness & Alert Type & R.A.(deg) & Decl.(deg) & Coincident 4LAC Source/Comments & $\begin{array}{c}\text { GCN } \\
\text { Circular }\end{array}$ \\
\hline$\overline{\text { IC-191001A }}$ & $58.9 \%$ & GOLD & $314.08_{-2.26}^{+6.56}$ & $12.94_{-1.47}^{+1.50}$ & Large angular uncertainty & 25913 \\
\hline IC-190922B & $50.5 \%$ & GOLD & $5.76_{-1.37}^{+1.19}$ & $-1.57_{-0.82}^{+0.93}$ & $\ldots$ & 25806 \\
\hline IC-190819A & $29.2 \%$ & BRONZE & $148.80_{-3.24}^{+2.07}$ & $1.38_{-0.75}^{+1.00}$ & Large angular uncertainty & 25402 \\
\hline IC-190730A & $67.2 \%$ & GOLD & $225.79_{-1.43}^{+1.28}$ & $10.47_{-0.89}^{+1.14}$ & 4FGL J1504.4+1029 & 25225 \\
\hline IC-190712A & $30.3 \%$ & BRONZE & $76.46_{-6.83}^{+5.09}$ & $13.06_{-3.44}^{+4.48}$ & Large angular uncertainty & 25057 \\
\hline IC-190629A & $33.9 \%$ & BRONZE & 27.22 & $84.33_{-3.13}^{+4.95}$ & Decl. value too close to pole for accurate error on R.A. & 24910 \\
\hline IC-190619A & $54.5 \%$ & GOLD & $343.26_{-2.63}^{+4.08}$ & $10.73_{-2.61}^{+1.51}$ & Large angular uncertainty & 24854 \\
\hline IC-190529A & $53 \%$ & HESE & $\ldots$ & $\ldots$ & Retracted & 24674 \\
\hline IC-190504A & $63 \%$ & HESE & 65.77 & -37.44 & $\begin{array}{l}\text { No detailed angular uncertainty provided (IceCube } \\
\text { Collaboration 2019b) }\end{array}$ & 24392 \\
\hline IC-190503A & $36.6 \%$ & EHE & $120.28_{-0.77}^{+0.57}$ & $6.35_{-0.70}^{+0.76}$ & $\ldots$ & 24378 \\
\hline IC-190104A & $35 \%$ & HESE & $357.98_{-2.1}^{+2.3}$ & $-26.65_{-2.5}^{+2.2}$ & $\ldots$ & 23605 \\
\hline IC-181031A & $87 \%$ & HESE & $\ldots$ & $\cdots$ & Retracted & 23398 \\
\hline IC-181023A & $28.0 \%$ & EHE & $270.18_{-1.70}^{+2.00}$ & $-8.57_{-1.30}^{+1.25}$ & Large angular uncertainty & 23375 \\
\hline IC-181014A & $10 \%$ & HESE & $225.15_{-2.85}^{+1.40}$ & $-34.80_{-1.85}^{+1.15}$ & Large angular uncertainty & 23338 \\
\hline IC-180908A & $34.4 \%$ & EHE & $144.58_{-1.45}^{+1.55}$ & $-2.13_{-1.2}^{+0.9}$ & $\ldots$ & 23214 \\
\hline IC-180423A & $34 \%$ & HESE & $\ldots$ & $\ldots$ & Retracted & 22669 \\
\hline IC-171106A & $74.6 \%$ & EHE & $340.00_{-0.50}^{+0.70}$ & $+7.40_{-0.25}^{+0.35}$ & $\cdots$ & 22105 \\
\hline IC-171028A & $30 \%$ & HESE & $\cdots$ & $\cdots$ & Retracted & 22065 \\
\hline IC-171015A & $51 \%$ & HESE & $162.86_{-1.70}^{+2.60}$ & $-15.44_{-2.00}^{+1.60}$ & Large angular uncertainty & 22016 \\
\hline IC-170922A & $56.5 \%$ & EHE & $77.43_{-0.80}^{+1.30}$ & $5.72_{-0.40}^{+0.70}$ & 4FGL J0509.4+0542 & 21916 \\
\hline IC-170506A & $35 \%$ & HESE & $\ldots$ & $\ldots$ & Consistent with atmospheric muon background & 21075 \\
\hline IC-170321A & $28.0 \%$ & EHE & $98.30_{-1.2}^{+1.2}$ & $-15.02_{-1.2}^{+1.2}$ & $\cdots$ & 20929 \\
\hline
\end{tabular}

Note. Coordinates are reported in J2000 epoch with $90 \%$ uncertainties. Alerts with a $90 \%$ angular error larger than 5 deg $^{2}$ are excluded from the analysis. For alerts shown in bold, a 4LAC source was identified located within the $90 \%$ uncertainty region. The signalness is added for completion but not used for further analysis. Alerts are taken from https://gcn.gsfc.nasa.gov/amon_hese_events.html, https://gcn.gsfc.nasa.gov/amon_ehe_events.html, and https://gcn.gsfc.nasa.gov/amon_ icecube_gold_bronze_events.html.

4FGL sources, 4FGL J1758.7-1621 and 4FGL J1750.4-1721. The first one is associated with a counterpart named AT20G J175841-161703 and classified as a blazar of uncertain type (BCU), while the second one is unassociated. The neutrino best-fit position is located just $4^{\circ}$ from the Galactic plane, where the source density is high and the large diffuse emission complicates the detection and association. Since our work focuses on AGNs, we only consider the BCU.

In this work, we identify two additional new coincidences with archival neutrino events using 4LAC compared to the ones reported in Garrappa et al. (2019), where 3LAC was used to search for coincidences. They are IC-150926A, spatially coincident with 4FGL J1258.7-0452, and IC-161103A, spatially coincident with 4FGL J0244.7+1316. Both sources are included in $4 \mathrm{LAC}$ but not in 3LAC.
The ANTARES collaboration (Aublin 2019) searched for an excess of neutrinos from the positions of 3LAC sources and reported a hot spot of ANTARES neutrinos from the direction of MG3 J225517+2409. The object MG3 J225517+2409 is also spatially coincident with the $340 \mathrm{TeV}$ neutrino IC100608A with 65\% signalness (event number three in Aartsen et al. 2016) and in flaring state during the IceCube neutrino arrival time (Aublin 2019). We note that IC-100608A would not have passed our selection criteria outlined above due to its large $90 \%$ angular uncertainty of roughly $30 \mathrm{deg}^{2}$ (assuming an elliptical shape). Therefore, the source was excluded from our source sample test presented in Section 4.2. However, the temporal coincidence with IC-100608A and spatial coincidence of the ANTARES hot spot make this source interesting as a potential counterpart. 
Table 2

Archival Neutrino Alerts

\begin{tabular}{|c|c|c|c|c|}
\hline IceCube Event Name & Alert Type & R.A.(deg) & Decl.(deg) & Coincident 4LAC Source/Comments \\
\hline IC-160510A & EHE & $352.34_{-1.31}^{+1.63}$ & $2.09_{-0.85}^{+0.99}$ & $\cdots$ \\
\hline IC-160128A & EHE & $263.40_{-1.18}^{+1.35}$ & $-14.79_{-1.02}^{+0.99}$ & $\cdots$ \\
\hline IC-151207A & HESE & $\cdots$ & $\cdots$ & Bad angular resolution would have been retracted \\
\hline IC-151122A & EHE & $262.18_{-1.21}^{+0.90}$ & $-2.38_{-0.43}^{+0.73}$ & $\ldots$ \\
\hline IC-150926A & EHE & $194.50_{-1.21}^{+0.76}$ & $-4.34_{-0.95}^{+0.70}$ & 4FGL J1258.7-0452 \\
\hline IC-150923A & EHE & $103.27_{-1.36}^{+0.70}$ & $3.88_{-0.71}^{+0.59}$ & $\cdots$ \\
\hline IC-150911A & HESE & $240.20_{-1.38}^{+1.29}$ & $-0.45_{-1.23}^{+1.17}$ & Large angular uncertainty \\
\hline IC-150831A & EHE & $54.85_{-0.98}^{+0.94}$ & $33.96_{-1.19}^{+1.07}$ & $\cdots$ \\
\hline IC-150812A & EHE & $328.19_{-1.03}^{+1.01}$ & $6.21_{-0.49}^{+0.44}$ & $\ldots$ \\
\hline IC-150428A & HESE & $80.77_{-1.23}^{+1.12}$ & $-20.75_{-0.83}^{+0.45}$ & $\ldots$ \\
\hline IC-141209A & HESE & $160.05_{-1.04}^{+0.84}$ & $6.57_{-0.56}^{+0.64}$ & 4FGL J1040.5+0617 \\
\hline IC-141109A & HESE & $55.63_{-1.53}^{+0.79}$ & $-16.50_{-0.68}^{+0.81}$ & No coincident sources \\
\hline IC-140923A & EHE & $169.72_{-0.86}^{+0.91}$ & $-1.34_{-0.66}^{+0.73}$ & $\ldots$ \\
\hline IC-140611A & EHE & $110.30_{-0.45}^{+0.66}$ & $11.57_{-0.24}^{+0.14}$ & $\ldots$ \\
\hline IC-140420A & HESE & $238.98_{-1.91}^{+1.81}$ & $-37.73_{-1.31}^{+1.47}$ & Large angular uncertainty \\
\hline IC-140203A & EHE & $349.54_{-1.97}^{+2.21}$ & $-13.71_{-1.38}^{+1.23}$ & Large angular uncertainty \\
\hline IC-140122A & HESE & $219.64_{-4.16}^{+5.16}$ & $-86.16_{-0.60}^{+0.55}$ & Large angular uncertainty \\
\hline IC-140109A & EHE & $292.85_{-0.94}^{+0.87}$ & $33.06_{-0.46}^{+0.50}$ & $\ldots$ \\
\hline IC-140108A & EHE & $344.53_{-0.48}^{+0.67}$ & $1.57_{-0.32}^{+0.35}$ & $\cdots$ \\
\hline IC-131204A & EHE & $289.16_{-0.94}^{+1.08}$ & $-14.25_{-0.81}^{+0.91}$ & $\ldots$ \\
\hline IC-131202A & HESE & $206.63_{-1.56}^{+2.04}$ & $-22.02_{-1.04}^{+1.69}$ & Large angular uncertainty \\
\hline IC-131023A & EHE & $301.82_{-0.93}^{+1.10}$ & $11.49_{-1.09}^{+1.19}$ & $\cdots$ \\
\hline IC-130907A & EHE & $129.81_{-0.28}^{+0.48}$ & $-10.36_{-0.31}^{+0.36}$ & $\cdots$ \\
\hline IC-130627A & HESE & $93.43_{-0.85}^{+0.80}$ & $14.02_{-0.75}^{+0.72}$ & No coincident sources \\
\hline IC-130408A & HESE & $167.17_{-1.90}^{+2.87}$ & $20.67_{-0.89}^{+1.15}$ & Large angular uncertainty \\
\hline IC-121011A & EHE & $205.22_{-0.65}^{+0.59}$ & $-2.39_{-0.57}^{+0.51}$ & $\ldots$ \\
\hline IC-120922A & EHE & $70.75_{-1.63}^{+1.56}$ & $19.79_{-0.68}^{+1.37}$ & Large angular uncertainty \\
\hline IC-120523A & EHE & $171.03_{-0.90}^{+0.81}$ & $26.36_{-0.30}^{+0.49}$ & $\cdots$ \\
\hline IC-120501A & HESE & $\ldots$ & $\ldots$ & Bad angular resolution would have been retracted \\
\hline IC-120301A & EHE & $238.01_{-0.59}^{+0.60}$ & $18.60_{-0.39}^{+0.46}$ & $\ldots$ \\
\hline IC-111228A & HESE & $\cdots$ & $\cdots$ & Bad angular resolution would have been retracted \\
\hline IC-110930A & EHE & $266.48_{-1.55}^{+2.09}$ & $-4.41_{-0.86}^{+0.59}$ & 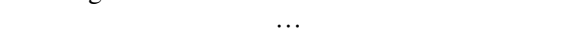 \\
\hline IC-110714A & HESE & $67.86_{-0.72}^{+0.51}$ & $40.32_{-0.25}^{+0.73}$ & $\cdots$ \\
\hline IC-110304A & EHE & $116.37_{-0.73}^{+0.73}$ & $-10.72_{-0.65}^{+0.57}$ & $\cdots$ \\
\hline IC-110216A & HESE & $\ldots$ & $\ldots$ & Bad angular resolution would have been retracted \\
\hline IC-110128A & EHE & $307.53_{-0.81}^{+0.82}$ & $1.19_{-0.32}^{+0.35}$ & $\cdots$ \\
\hline IC-101112A & HESE & $110.56_{-0.37}^{+0.80}$ & $-0.37_{-0.65}^{+0.48}$ & $\cdots$ \\
\hline IC-101028A & EHE & $88.68_{-0.55}^{+0.54}$ & $0.46_{-0.27}^{+0.33}$ & $\cdots$ \\
\hline IC-101009A & EHE & $331.09_{-0.72}^{+0.56}$ & $11.10_{-0.58}^{+0.48}$ & $\cdots$ \\
\hline IC-100912A & HESE & $\ldots$ & $\ldots$ & Bad angular resolution would have been retracted \\
\hline
\end{tabular}

Note. Coordinates are reported in J2000 epoch with $90 \%$ uncertainties. Alerts with a 90\% angular uncertainty larger than 5 deg $^{2}$ are excluded from the analysis. For alerts shown in bold, a 4LAC source was identified located within the $90 \%$ uncertainty region. The signalness is added for completion but not used for further analysis. Archival events are taken from https://icecube.wisc.edu/science/data/TXS0506_alerts.

Kun et al. (2017) reported a spatial coincidence between the HESE event IC-101112A and flat-spectrum radio quasar (FSRQ) PKS 0723-008. We do not consider this source here because it lies outside the reported $90 \%$ uncertainty region, ${ }^{11}$ which is smaller than the originally published uncertainty radius in Aartsen et al. (2014).

\section{Multiwavelength Data}

In the following, we motivate why different wavelengths may provide relevant information connected to high-energy neutrino emission.

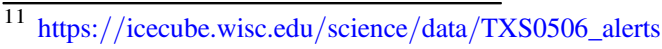

High-energy neutrinos are produced together with highenergy photons of similar energy in hadronic processes. The $\mathrm{TeV}$ to PeV photons are quickly absorbed within the source or in interactions with the extragalactic background light through photon-photon annihilation and cascade down to lower energies. Hence, GeV gamma-rays detected by Fermi-LAT provide the all-sky data set closest in energy to the neutrinos of interest. However, if the source environment is optically thick to $\mathrm{GeV}$ gamma-rays due to high densities of photons in the $\mathrm{keV}$ range, then those gamma-rays will cascade down to even lower energies, which then become an important tracer of the source activity as well. Furthermore, $\mathrm{TeV}$ instruments relying on the imaging atmospheric Cerenkov technique have a limited field of view and therefore even with archival data do not provide 
all-sky coverage. All-sky TeV instruments such as HAWC (Abeysekara et al. 2013) have limited sensitivity to extragalactic sources due to extragalactic background light (EBL) absorption.

It is possible that X-rays would be a good tracer for hadronic interactions in sources where the $\mathrm{GeV}$ emission is dominated by leptonic processes (Keivani et al. 2018; Gao et al. 2019).

Increased radio emission was found from TXS $0506+056$ at the arrival time of IC-170922A and PKS B1424-418 in coincidence with the arrival time of a $\mathrm{PeV}$ neutrino (Kadler et al. 2016). We note that the chance coincidence of the neutrino association with the latter was relatively large $(5 \%)$. Britzen et al. (2019) used radio data to suggest a possible collision of two jets in TXS 0506+056. However, Ros et al. (2020) excluded the presence of a secondary jet core with higher-resolution radio data but found signs of a spine-sheath structure of the jet, which could be relevant for neutrino production (see also Ghisellini et al. 2005; Tavecchio et al. 2014; Ansoldi et al. 2018).

Gamma-ray and X-ray polarization data could be used to pinpoint the leptonic and/or hadronic blazar radiation mechanisms in the high-energy bands and infer the magnetic field strength in the emission region (Zhang et al. 2019), but they are not available for the sources in our sample.

Finally, archival optical data are available for all sources in our sample. In combination with gamma-ray data, optical data can be useful to identify high-energy flares without low-energy counterparts, which could be due to hadronic interaction (Krawczynski et al. 2004).

\subsection{Fermi-LAT Data}

The Fermi-LAT is a pair-conversion telescope sensitive to gamma-rays with energies from $20 \mathrm{MeV}$ to greater than 300 $\mathrm{GeV}$ (Atwood et al. 2009). It has a field of view $>2 \mathrm{sr}$ and scans the entire sky every $3 \mathrm{hr}$ during standard operations. We use almost 11 yr of Pass 8 data collected between 2008 August 4 and 2019 May 30 (MJD 54,682-58,633) with an exception for the source PKS $1502+106$, for which we use data up to 2019 July 31 (MJD 58,695) in order to include the arrival time of IC-190730A. We select photons from the event class developed for point-source analyses ${ }^{12}$ in the energy range from $100 \mathrm{MeV}$ to $800 \mathrm{GeV}$ binned into 10 logarithmically spaced energy intervals per decade. We select a region of interest (ROI) of $15^{\circ} \times 15^{\circ}$ centered on the gamma-ray source position, binned in 0.1 size pixels. The binning is applied in celestial coordinates using a Hammer-Aitoff projection. We perform a maximum-likelihood analysis using the standard Fermi-LAT ScienceTools package version v11r04p00 available from the Fermi Science Support Center ${ }^{13}$ (FSSC) and the P8R3_SOURCE_V2 instrument response functions, together with the fermipy package v0.17.4 (Wood et al. 2017).

We use standard data-quality cuts to select events observed when the detector was in a normal operation mode. In order to obtain a sample of events for each analysis with reduced contamination from gamma-rays produced in the Earth's upper atmosphere, we apply an additional instrument zenith angle cut of $\theta<90^{\circ}$. We also remove time periods coinciding with bright solar flares and gamma-ray bursts detected by the LAT.

\footnotetext{
${ }^{12}$ http://fermi.gsfc.nasa.gov/ssc/data/analysis/documentation/Pass8_ usage.html

${ }^{13}$ http://fermi.gsfc.nasa.gov/ssc/data/analysis/
}

The input model for the ROI includes all known gamma-ray sources from the 4FGL catalog in a region of $20^{\circ} \times 20^{\circ}$, slightly larger than the ROI, and the isotropic and Galactic diffuse gamma-ray emission models provided by the standard templates iso_P8R3_SOURCE_V2_v01.txt and gll_iem_v07. fits. ${ }^{14}$ The effect of energy dispersion is included in the fits performed with the Fermi-LAT ScienceTools for all point sources and the Galactic diffuse gamma-ray emission model. We use an iterative source-finding algorithm to scan the ROI and include in the model sources that are significantly $(\geqslant 5 \sigma)$ detected over the full data set time range but not over the $8 \mathrm{yr}$ data that produced the 4FGL catalog. New putative point sources are modeled with a single power-law spectrum with the index fixed to 2 and the normalization free to vary in the fit. The search procedure is iterated until no further significant excess is found. The new point sources significantly detected in the longer-integration time data set are accounted for by the final ROI model.

The definition of test statistics (TS) from Mattox et al. (1996) is used to measure the detection level of each source. The minimum separation allowed between two independent pointsource detections is set to 0.3 . We compute the light curve for each source using the adaptive binning algorithm from Lott et al. (2012) with the prescriptions outlined in Garrappa et al. (2019), in order to better resolve the flaring activities of the target sources. Statistically significant variations in the light curve's behavior are detected in this work with the Bayesian Blocks algorithm (Scargle et al. 2013), for which we use its Astropy implementation. ${ }^{15} \mathrm{We}$ adopt a prior that makes the algorithm sensitive to variations that are significant at a $95 \%$ confidence level.

All reported gamma-ray fluxes are in the analysis energy range from $100 \mathrm{MeV}$ to $800 \mathrm{GeV}$.

\subsection{Neil Gehrels Swift Observatory Data}

While no sensitive all-sky X-ray monitor exists, we can take advantage of the pointed observations of the Swift X-Ray Telescope (XRT) collected in target-of-opportunity and monitoring operations. We first reprocessed the Swift-XRT data to calibrate and clean the event files using the task xrtpipeline.

The pipeline xrtgrblc was adopted to extract the source and background spectra and ancillary response files used for the light-curve generation. This tool automatically adjusts the source and background region sizes based on the source count rate. ${ }^{16}$ Due to the low photon statistics of the individual observation IDs, we fit a simple absorbed power-law model in XSPEC (Arnaud 1996) while taking the Galactic neutral hydrogen column density along the line of sight from Kalberla et al. (2005).

For the broadband SEDs, the event files were combined with xselect. Exposure maps and ancillary response files were extracted with the tasks ximage and xrtmkarf. The source region was chosen as a circle of $47^{\prime \prime}$ radius centered at the target, whereas the background region has an annular shape with inner and outer radii of $70^{\prime \prime}$ and $150^{\prime \prime}$, respectively, centered at the source of interest. We tested both an unbroken and a broken power law, taking into account the Galactic

\footnotetext{
${ }_{14}$ https://fermi.gsfc.nasa.gov/ssc/data/access/lat/BackgroundModels.html

15 http://docs.astropy.org/en/stable/api/astropy.stats.bayesian_blocks.html

16 https://heasarc.gsfc.nasa.gov/lheasoft/ftools/headas/xrtgrblc.html
} 
neutral hydrogen column density along the line of sight (Kalberla et al. 2005), and report the spectral parameters for the model that represents the data better. Depending on the source brightness, the source spectra are rebinned to have at least one or 20 counts per bin $^{-1}$. The spectral analysis is performed in XSPEC.

Snapshot observations from the UltraViolet and Optical Telescope (UVOT) on board the Swift satellite during each pointing to the target source are first combined using the tool uvotimsum. To derive the source instrumental magnitude using uvotsource, we adopt a circular source region of $5^{\prime \prime}$ radius centered at the object position, and a nearby source-free region of $30^{\prime \prime}$ radius is considered to derive the background contamination. The computed magnitudes are converted to energy flux units using the zero-points and calibrations of Breeveld et al. (2011) corrected for the Galactic reddening following Schlafly \& Finkbeiner (2011).

\subsection{ASAS-SN and CSS Optical Data}

Optical data in the $V$ and $g$ bands from the All-Sky Automated Survey for Supernovae (ASAS-SN; Shappee et al. 2014; Kochanek et al. 2017) are processed by the fully automatic ASAS-SN pipeline using the ISIS image subtraction package (Alard \& Lupton 1998; Alard 2000). We then perform aperture photometry on the subtracted science image using the IRAF apphot package, adding back in the flux from the reference image. The photometry is calibrated using the AAVSO Photometric All-Sky Survey (APASS; Henden et al. 2015).

Additional $V$-band data from the Catalina Sky Survey (CSS; Drake et al. 2009) are available from the public database and based on aperture photometry. To mitigate color-dependent differences between the UVOT, CSS, and ASAS-SN $V$-band filters, we add an offset to the ASAS-SN and UVOT data to match the CSS data in regions with overlapping exposure. A similar offset was applied to the ASAS-SN $g$-band observations to line them up with the $V$-band data. The applied shift is a constant in flux space and indicated in the legend of the corresponding light-curve figures. Since our study only relies on the shape of the light curve rather than the absolute optical flux level, and given that none of the neutrino flares occurred in the transition region between ASAS-SN and CSS data, this shift is not critical for our results.

\subsection{Radio Data}

Owens Valley Radio Observatory (OVRO) $15 \mathrm{GHz}$ radio monitoring data (Richards et al. 2011) are available for nine sources of the sample (one of them is TXS 0506+056, which was already presented in Aartsen et al. 2018a).

\subsection{Other Data}

We collect archival spectral observations with the Space Science Data Center SED builder tool ${ }^{17}$ to supplement the data analyzed in this work. This allows us to cover the broadband SED of the target objects as well as possible, admittedly using nonsimultaneous data sets. However, considering that these observations represent the "average" activity of the sources, we can use them to compare the existing data acquired contemporaneously to the reported neutrino events.

\footnotetext{
17 https://tools.ssdc.asi.it/
}

\section{Methods}

\subsection{Quantifying the Gamma-Ray Activity during the Neutrino Arrival Time}

For each individual source, we calculate the chance probability

$$
p_{\gamma}\left(F_{\gamma, \nu}\right)=\frac{\int_{-\infty}^{\infty}\left(\sum_{i} t_{i} \int_{F_{x}}^{\infty} \mathcal{N}\left(x, F_{i}, \sigma_{i}\right) d x\right) \mathcal{N}\left(F_{x}, F_{\gamma, \nu}, \sigma_{\gamma, \nu}\right) d F_{x}}{\sum_{i} t_{i}}
$$

to find the neutrino in a period of gamma-ray activity larger than the gamma-ray energy flux $F_{\gamma, \nu}$ in the time bin $t$ overlapping with the neutrino arrival. Here $\mathcal{N}$ is a Gaussian function with mean $F_{\gamma, \nu}$ and standard deviation $\sigma_{\gamma, \nu}$ evaluated at $F_{x}$; i.e., we assume that the flux uncertainty is normally distributed. The index $i$ runs over all time bins of the source of interest, and the $p_{\gamma}$ for all sources is reported in Table 3. Low values of $p_{\gamma}$ indicate that the source was in a high gamma-ray flux state during the neutrino arrival time compared to the other time bins in the $11 \mathrm{yr}$ light curve, while high values indicate that the source did not show an excess in gamma-rays in temporal coincidence with the neutrino emission. We use the adaptive bins that were used to compile the gamma-ray light curves. Due to noncontinuous exposure and gaps in the data, we do not perform a similar analysis for optical and X-ray data. We note that the optical data show in general a similar temporal behavior to the gamma-ray data, as was found in previous studies (see, e.g., Cohen et al. 2014).

\subsection{Comparison of Neutrino Blazar Candidates to the Gamma-Ray Blazar Sample}

In addition to studying the multiwavelength behavior of individual sources, we study the average gamma-ray properties of the sources identified as potential neutrino emitters and compare them to the entire gamma-ray blazar population. Figure 1 shows the time-integrated gamma-ray energy flux in the energy range from $100 \mathrm{MeV}$ to $100 \mathrm{GeV}$ as a function of redshift for all blazars in 4LAC (including BCUs). All values are taken from 4LAC. We have added the redshift of four sources (see Table 3). We apply a Kolmogorov-Smirnov (K-S) test to determine how compatible the gamma-ray energy flux distribution of the candidate neutrino blazars is with the expected distribution of gamma-ray blazars under a given hypothesis. To verify that the $\mathrm{K}-\mathrm{S}$ test $p$-value is not biased, we performed a sanity check with randomized data. We generate a background K-S $p$-value distribution by randomly selecting $N$ blazars from the entire blazar sample and calculating the K-S $p$ value for those. Here $N$ is the number of identified neutrino blazar candidates. A calibrated $p$-value for the measurement is then calculated as the ratio of background $p$-values smaller than the measured K-S $p$-value. We note that no significant bias was found, and the calibrated $p$-value is similar to the one obtained directly from the K-S test method.

We compare the observed gamma-ray energy flux of candidate neutrino blazars to the expectation for three separate scenarios. First, we test the uncorrelated case, in which all neutrino blazar coincidences occur by chance. In that case, we expect the gamma-ray flux of the candidate neutrino blazars to follow the distribution of the gamma-ray blazar population as a 
Table 3

Neutrino Source Candidates

\begin{tabular}{|c|c|c|c|c|c|c|c|c|}
\hline Source Name & 4FGL Name & Class & Redshift & $T_{0}(\mathrm{MJD})$ & $T_{w}$ (days) & $p_{\gamma}$ & $T_{\gamma, \nu}(\mathrm{MJD})$ & $L_{\gamma}\left(\operatorname{erg~s}^{-1}\right)$ \\
\hline MG3 J225517+2409 & $\mathrm{J} 2255.2+2411$ & $\mathrm{BL} \mathrm{Lac}$ & $1.37^{\mathrm{a}}$ & $55,355.49$ & $\ldots$ & 0.04 & {$[55,346.73,55,403.54]$} & $1.3 \times 10^{47}$ \\
\hline 1RXS J125847.7-044746 & $\mathrm{J} 1258.7-0452$ & BL Lac & $0.586^{\mathrm{c}}$ & $57,291.90119$ & $\cdots$ & $\cdots$ & $\cdots$ & $2.9 \times 10^{45}$ \\
\hline GB6 J0244+1320 & $\mathrm{J} 0244.7+1316$ & $B C U^{d}$ & $\cdots$ & $57,695.38$ & $\cdots$ & $\cdots$ & $\cdots$ & $\cdots$ \\
\hline TXS 0506+056 & J0509.4+0542 & $\mathrm{BL} \mathrm{Lac} \mathrm{e}^{\mathrm{e}}$ & $0.336^{\mathrm{f}}$ & $58,018.87$ & $\ldots$ & 0.009 & {$[58,016.57,58,019.94]$} & $2.2 \times 10^{46}$ \\
\hline \multicolumn{9}{|c|}{ Neutrino Flare Candidates } \\
\hline $4 C+20.25$ & $\mathrm{~J} 1125.9+2005$ & FSRQ & 0.133 & $56,464.1$ & 5.2 & 0.64 & {$[56,369.45,57,248.31]$} & $1.6 \times 10^{44}$ \\
\hline CRATES J112916+370317 & $\mathrm{J} 1129.1+3703$ & $\mathrm{BL} \mathrm{Lac}$ & 0.445 & $56,501.385$ & $6.0 \times 10^{-2}$ & 0.45 & {$[56,404.68,57,066.59]$} & $2.9 \times 10^{46}$ \\
\hline MG2 J112758+3620 & $\mathrm{J} 1127.8+3618$ & FSRQ & 0.884 & $56,501.385$ & $6.0 \times 10^{-2}$ & 0.24 & {$[56,482.90,56,555.93]$} & $5.5 \times 10^{46}$ \\
\hline TXS $0506+056$ & J0509.4+0542 & $\mathrm{BL} \mathrm{Lac}{ }^{\mathrm{e}}$ & 0.336 & 57,000 & 120 & 0.92 & {$[56,965.28,57,089.28]$} & $2.2 \times 10^{46}$ \\
\hline GB6 J0929+5013 & J0929.3+5014 & BL Lac & $0.37^{\mathrm{h}}$ & $57,758.0$ & 1.2 & 0.44 & {$[57,647.78,57,759.66]$} & $5.6 \times 10^{45}$ \\
\hline 1ES $0927+500$ & J0930.5+4951 & BL Lac & 0.187 & $57,758.0$ & 1.2 & 0.49 & {$[57,031.36,58,633.01]$} & $2.2 \times 10^{44}$ \\
\hline
\end{tabular}

Notes. The first seven sources were found in coincidence with single high-energy neutrinos, while the remaining sources were found coincident with neutrino flares by O'Sullivan \& Finley (2019). The source classes and redshifts (if not noted otherwise) are reported in the 4LAC catalog (Fermi-LAT Collaboration 2019). Here $T_{0}$ is the central time of the reported neutrino flare, $T_{w}$ is twice the standard deviation of the Gaussian flare, $p_{\gamma}$ is the probability that the neutrino flare center is coincident with a gamma-flare of the found or larger flux, and $T_{\gamma, \nu}$ is the time window used to calculate the gamma-ray flux in which the neutrino arrived. The gamma-ray luminosity is the $8 \mathrm{yr}$ average calculated from the 4LAC values. Luminosity is only calculated when a redshift measurement is available. The objects $1 \mathrm{RXS}$ J125847.7-044746 and GB6 J0244+1320 are too dim in gamma-rays to study the variability.

a Redshift from 4LAC, which is taken from SDSS, where it is flagged as "chi-squared of best fit is too close to that of second best ( $<0.01$ in reduced chi-squared)." Paiano et al. (2019) found that the redshift is $>0.8633$.

${ }^{\mathrm{b}}$ Redshift from Ahn et al. (2012).

${ }^{c}$ Redshift from Bauer et al. (2000).

${ }^{\mathrm{d}}$ Blazar of uncertain type.

e Note that TXS 0506+056 was reclassified by Padovani et al. (2019) as "masquerading BL Lac," i.e., intrinsically an FSRQ with hidden broad lines and a standard accretion disk.

${ }^{\mathrm{f}}$ Redshift from Paiano et al. (2018).

${ }^{\mathrm{g}}$ Narrow-line Seyfert 1.

${ }^{\mathrm{h}}$ Redshift from Abazajian et al. (2009).

whole. Second, we test the hypothesis of a linear correlation of the neutrino flux with the gamma-ray energy flux of blazars. In that case, we expect the candidate neutrino blazars to have preferentially higher gamma-ray energy flux. In the third case, we test whether the neutrino flux is proportional to the square of the gamma-ray energy flux, as has been suggested in Oikonomou et al. (2019). Here we expect the candidate neutrino blazar distribution to be skewed toward even higher gamma-ray energy fluxes.

For internal consistency, the single high-energy neutrino blazar candidates are compared to the 4LAC blazar population, while the neutrino flare blazar candidates are compared to 3LAC, because only $3 \mathrm{LAC}$ source positions were searched for neutrino flares in O'Sullivan \& Finley (2019). A large K-S pvalue implies that the data are well described by a given hypothesis, while a small one indicates that that hypothesis is disfavored.

The same K-S test is also applied to the candidate neutrino flare sources. As pointed out in O'Sullivan \& Finley (2019), one pair and one triplet of sources are correlated. After associating 4FGL J1129.1+3703 with CRATES J112916 +370317 , the triplet becomes a pair, because the second possible counterpart can be discarded. The position of
CRATES J112916+370317 is correlated with MG2 J112758 +3620 , which is associated with the gamma-ray source 4FGL $\mathrm{J} 1127.8+3618$. The second correlated source positions are GB6 $\mathrm{J} 0929+5013$ and 1ES 0927+500 (associated with the gammaray sources 4FGL J0929.3+5014 and 4FGL J0930.5+4951, respectively). We recalculate the K-S test using only one of the correlated source positions and quote a range of $p$-values, which brackets the outcome of removing a different set of sources from the test.

Because MG3 J225517+2409 did not fulfill our angular uncertainty criteria, it was excluded from the K-S test.

The results of the K-S test are presented in Table 4 and split into BL Lacs, FSRQs, and all blazars (including BCUs) combined.

\section{Results}

\subsection{Individual Sources}

The collected multiwavelength light curves are presented in multipanel Figures in the Appendix. We do not show the light curves of TXS 0506+056 and GB6 J1040+0617, because they were already discussed in detail in Garrappa et al. (2019). 


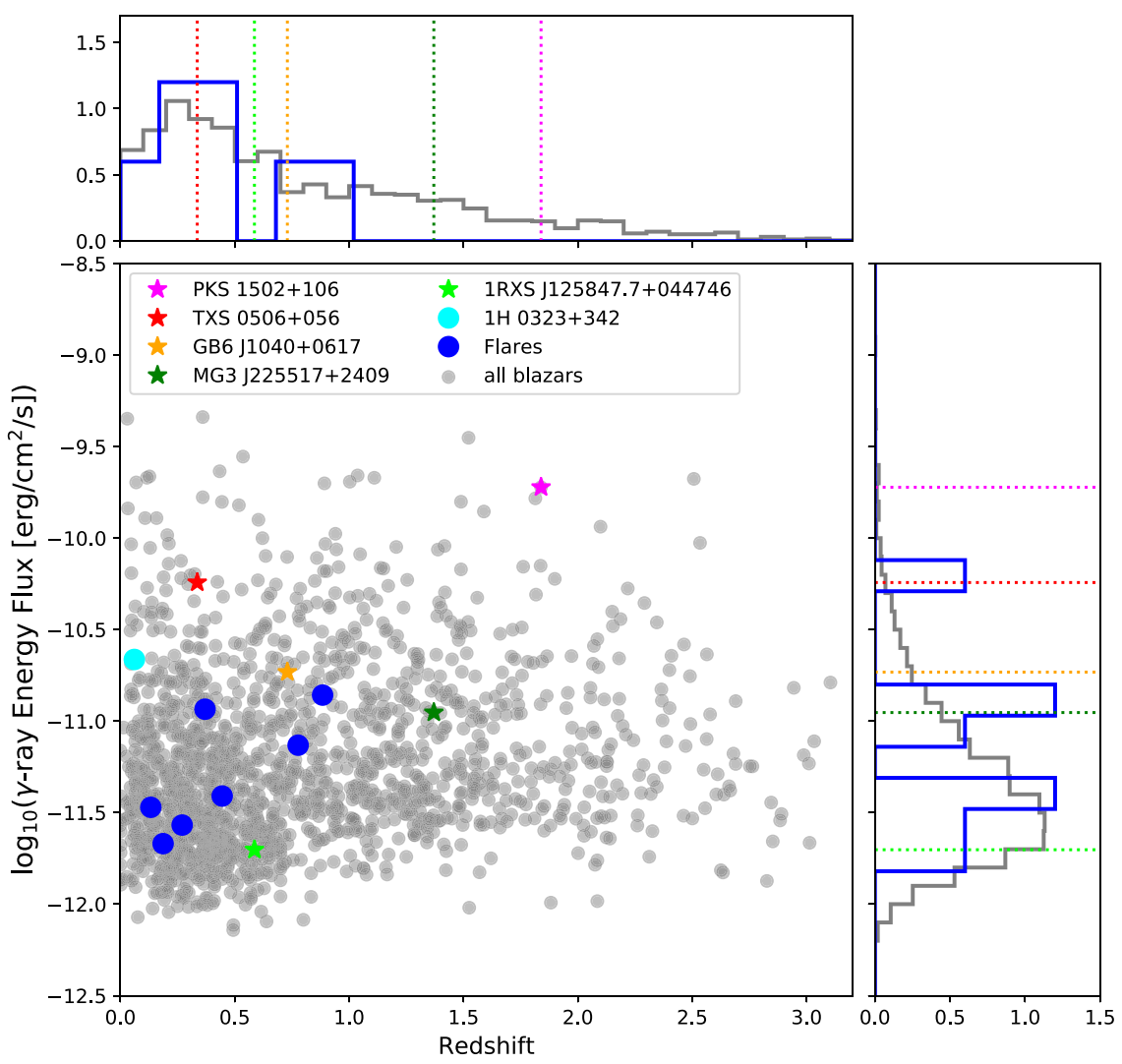

Figure 1. Comparison of candidate neutrino blazars with all blazars in the 4LAC AGN sample (shown in gray). The gamma-ray energy flux is shown as a function of redshift. Sources identified in the neutrino flare search are displayed with blue circles. The object $1 \mathrm{H} 0323+342$ is highlighted in cyan. Sources associated with single high-energy neutrinos are marked by colored stars. The side panels show projections of the distributions. The dashed lines in the projection panels are the values of individual blazars associated with single high-energy neutrinos, and the blue distribution shows the histogram of the neutrino flare candidate sources.

Table 4

K-S Test $p$-values

\begin{tabular}{|c|c|c|c|c|c|c|}
\hline & \multicolumn{2}{|c|}{ BL Lacs } & \multicolumn{2}{|c|}{ FSRQs } & \multicolumn{2}{|c|}{ All Blazars } \\
\hline & Uncorrelated & Correlated & Uncorrelated & Correlated & Uncorrelated & Correlated \\
\hline Single neutrinos & 0.32 & $0.45(0.0013)$ & 0.10 & $0.36(0.28)$ & 0.126 & $0.64(0.00032)$ \\
\hline Neutrino flares & $0.37-0.98$ & $0.027-0.533$ & $0.01-0.36$ & $0.0075-0.023$ & $0.39-0.98$ & $0.0039-0.021$ \\
\hline
\end{tabular}

Note. The range of $p$-values for the neutrino flare case comes from removing different combinations of the correlated source positions. Different columns represent the uncorrelated and linearly correlated hypothesis; values in parentheses represent the quadratically correlated case. Note that neutrino flare candidate blazars are compared to the 3LAC population and single high-energy neutrino candidate blazars with the 4LAC population.

All sources are detected in $\mathrm{GeV}$ gamma-rays, which is expected, since they are selected from the 3LAC or 4FGL catalog. However, some of them are too faint to resolve temporal structure. We present both the flux and spectral index variations assuming a power-law spectrum for the source in each bin.

Most sources have good coverage in the optical during the neutrino arrival times. Radio data from the OVRO monitoring program are available for nine out of 14 sources. The X-ray data are sparse and only available for eight sources. Only $1 \mathrm{H}$ $0323+342$ has good coverage in X-rays during the neutrino flare.

In the following, we discuss the three most interesting sources. We discuss the brightest source in gamma-rays, PKS $1502+106$, and the two sources, $1 \mathrm{H} 0323+342$ and MG3 $\mathrm{J} 225517+2409$, that show gamma-ray flares during the neutrino arrival time, reflected by small $p_{\gamma}$ of $8 \%$ and $4 \%$, respectively, while the other sources showed $p$-values ranging from $17 \%$ to $92 \%$. However, given that we have performed this calculation for 15 sources, these findings are well compatible with the background expectations.

\subsection{1. $1 \mathrm{H} 0323+342$}

The radio-loud narrow-line Seyfert 1 galaxy $1 \mathrm{H} 0323+342$ at $z=0.061$ (Zhou et al. 2007; Abdo et al. 2009) shows increased gamma-ray activity during the reported neutrino flare time (see Figure 2). The gamma-ray counts map integrated over $11 \mathrm{yr}$ of data is shown in Figure 3. The neutrino arrived during a mild excess in gamma-rays of $F_{\gamma}^{\text {peak }}=(2.8 \pm 0.7) \times$ $10^{-7} \mathrm{ph} \mathrm{cm}^{-2} \mathrm{~s}^{-1}$ and roughly 1 month after a flare in the $\mathrm{X}$-ray, UV, and optical (see Figure 2). The chance probability to find the neutrino in a period of increased gamma-ray activity at the level of $F_{\gamma}^{\text {peak }}$ or higher is $p_{\gamma}=8 \%$. The neutrino flare arrives in the time bin just next to the peak. We note that the 

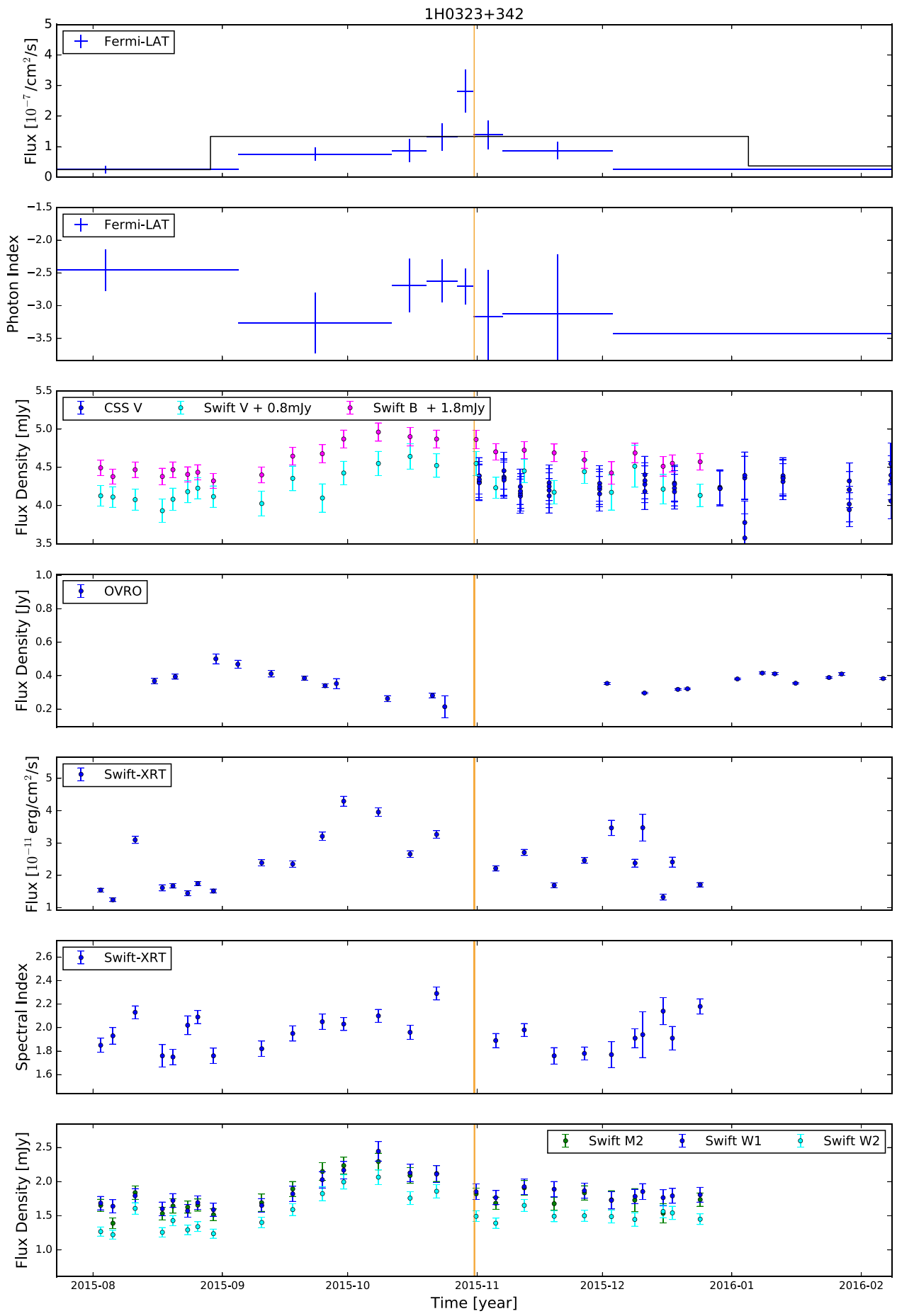

Figure 2. Multiwavelength light curve of $1 \mathrm{H} 0323+342$. The duration of the neutrino flare is short $\left(T_{w}=147 \mathrm{~s}\right)$, and its arrival time is shown as an orange line. An excess in gamma-rays is found coincident with the neutrino arrival time, and an excess in X-ray emission is visible roughly 1 month before the neutrino arrival time. The Fermi-LAT gamma-ray light curve covers the energy range from $100 \mathrm{MeV}$ to $800 \mathrm{GeV}$, the Swift X-ray light curve is from 0.3 to $10 \mathrm{keV}$, and the OVRO radio data are at $15 \mathrm{GHz}$.

source shows even stronger flares at earlier times, which are not found connected to neutrino emission.

Figure 4 (upper left panel) shows the broadband SED of $1 \mathrm{H}$ $0323+342$.

The X-ray spectrum reveals a break at $\sim 3 \mathrm{keV}$. The spectrum before the break energy is soft $\left(\Gamma_{1} \sim 2\right.$; see Table 5), possibly due to coronal emission (Abdo et al. 2009; Paliya et al. 2014, 2019). Note that various spectral features are detected in the X-ray spectrum of this source, which includes a soft X-ray excess below $2 \mathrm{keV}$, an Fe K-alpha emission line at $\sim 6 \mathrm{keV}$, and a possible Compton hump at higher frequencies (see, e.g., Paliya et al. 2014, 2019; Ghosh et al. 2018; Kynoch et al. 2018 for details). Covering these aspects is beyond the scope of this work. Furthermore, the broadband SED modeling of this object suggests that the gamma-ray emission region lies well within the BLR, i.e., close to the central black hole (Abdo et al. 2009; Paliya et al. 2014; Kynoch et al. 2018). If so, the $\mathrm{X}$-ray photons from the corona could constitute a target photon 

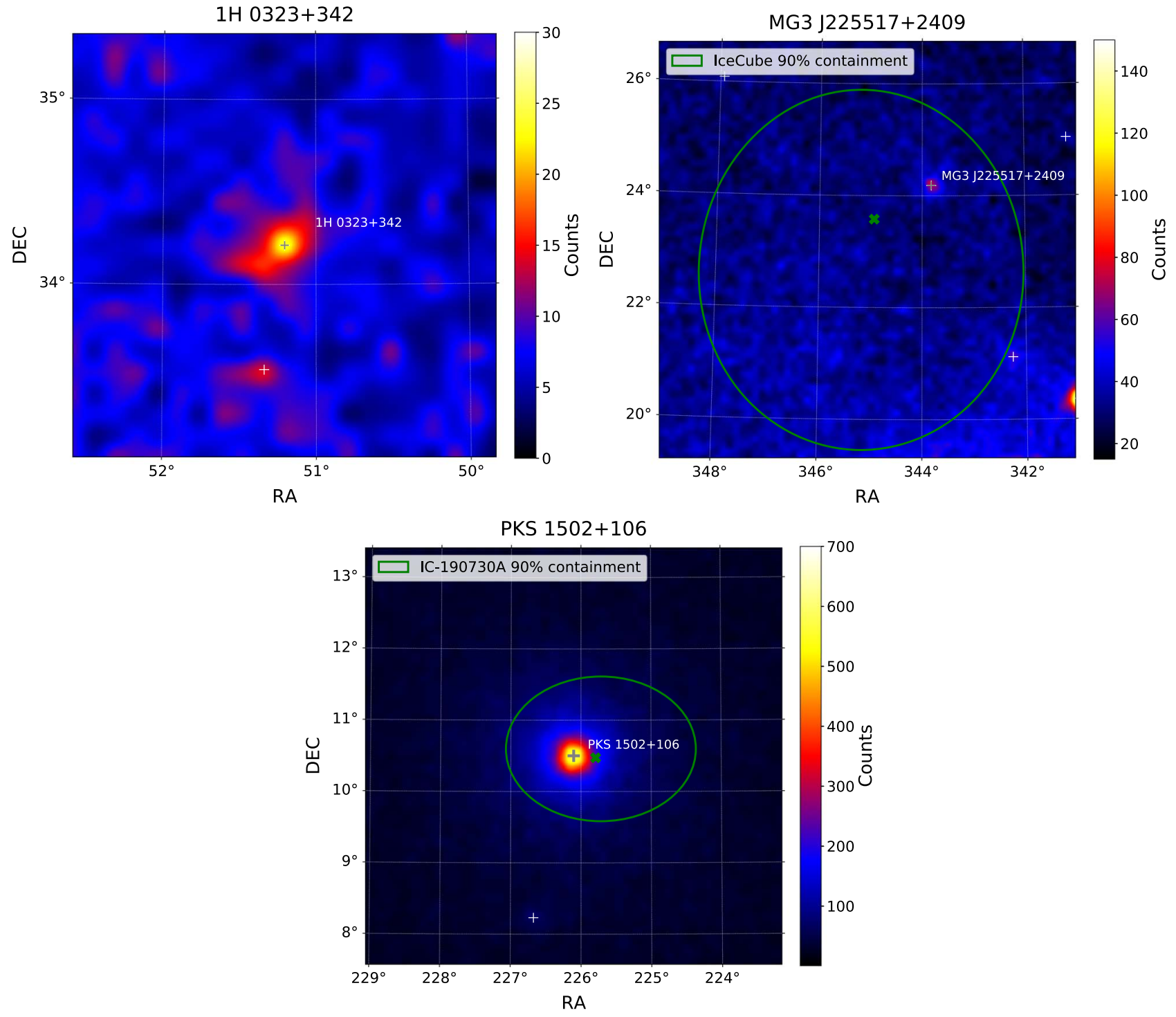

Figure 3. Gamma-ray count maps of $1 \mathrm{H} 0323+342$, MG3 J225517+2409, and PKS 1502+106 integrated over 11 yr of Fermi-LAT data. The green cross and green line show the best-fit neutrino position and 90\% uncertainty, respectively. White plus signs are 4FGL sources included in the background model. The count maps cover the energy range of $100 \mathrm{MeV}$ to $800 \mathrm{GeV}$, except for $1 \mathrm{H} 0323+342$, where we start at $1 \mathrm{GeV}$ to suppress the significant Galactic diffuse emission at the source's latitude of $b=-18.7$. The $1 \mathrm{H} 0323+342$ count map is not overlaid with a neutrino contour, since it was identified in the neutrino flare search from 3LAC sources; i.e., the neutrino flare candidate is by definition located at the position of $1 \mathrm{H} 0323+342$.

field for photohadronic interactions producing high-energy neutrinos. In particular, the interaction of the protons with the thermal continuum with a characteristic temperature $\left(T^{*}\right)$ can produce neutrinos with energy $E_{\nu} \sim 100 \mathrm{TeV}\left(T^{*} / 10^{5} \mathrm{~K}\right)^{-1}$ (see, e.g., Rodrigues et al. 2019). The X-ray coronal photons would also absorb the gamma-rays via pair production, leading to the steepening of the gamma-ray spectrum that is observed (Figure 4; Ghisellini \& Tavecchio 2009; Paliya et al. 2014; Rodrigues et al. 2019). Another observational signature for this process is the detection of a bright X-ray emission with a soft spectral shape (see Ghisellini \& Tavecchio 2009 for details), which is reflected in the X-ray spectrum of $1 \mathrm{H} 0323+342$ (Figure 4). A quantitative discussion will be the subject of a separate publication.

\subsection{2. $M G 3 J 225517+2409$}

The distant BL Lac object MG3 J225517+2409 shows a major flare coincident with the neutrino arrival time (see
Figure A1). A redshift of 1.37 (Fermi-LAT Collaboration 2019), which is taken from SDSS, is reported by 4LAC. However, the extracted redshift is flagged as "chi-squared of best fit is too close to that of second best $(<0.01$ in reduced chisquared)." Paiano et al. (2019) found that the redshift is $>0.8633$. The gamma-ray flare reaches a flux level of $(3.5 \pm 1.0) \times 10^{-8} \mathrm{ph} \mathrm{cm}^{-2} \mathrm{~s}^{-1}$ and lasts roughly 140 days (see Figure A1). The chance probability to find the neutrino in a period of increased gamma-ray activity at this level or higher is $p_{\gamma}=4 \%$.

Figure 4 (upper right panel) shows the broadband SED of MG3 J225517+2409, and the best-fit spectral values for the gamma-ray, X-ray, and UV bands are provided in Table 5.

\subsubsection{PKS $1502+106$}

The FSRQ PKS $1502+106$ was found to be located within the $50 \%$ uncertainty region of IC-190730A. The neutrino was reported with a signalness of $67 \%$ and an energy of $300 \mathrm{TeV}$ 

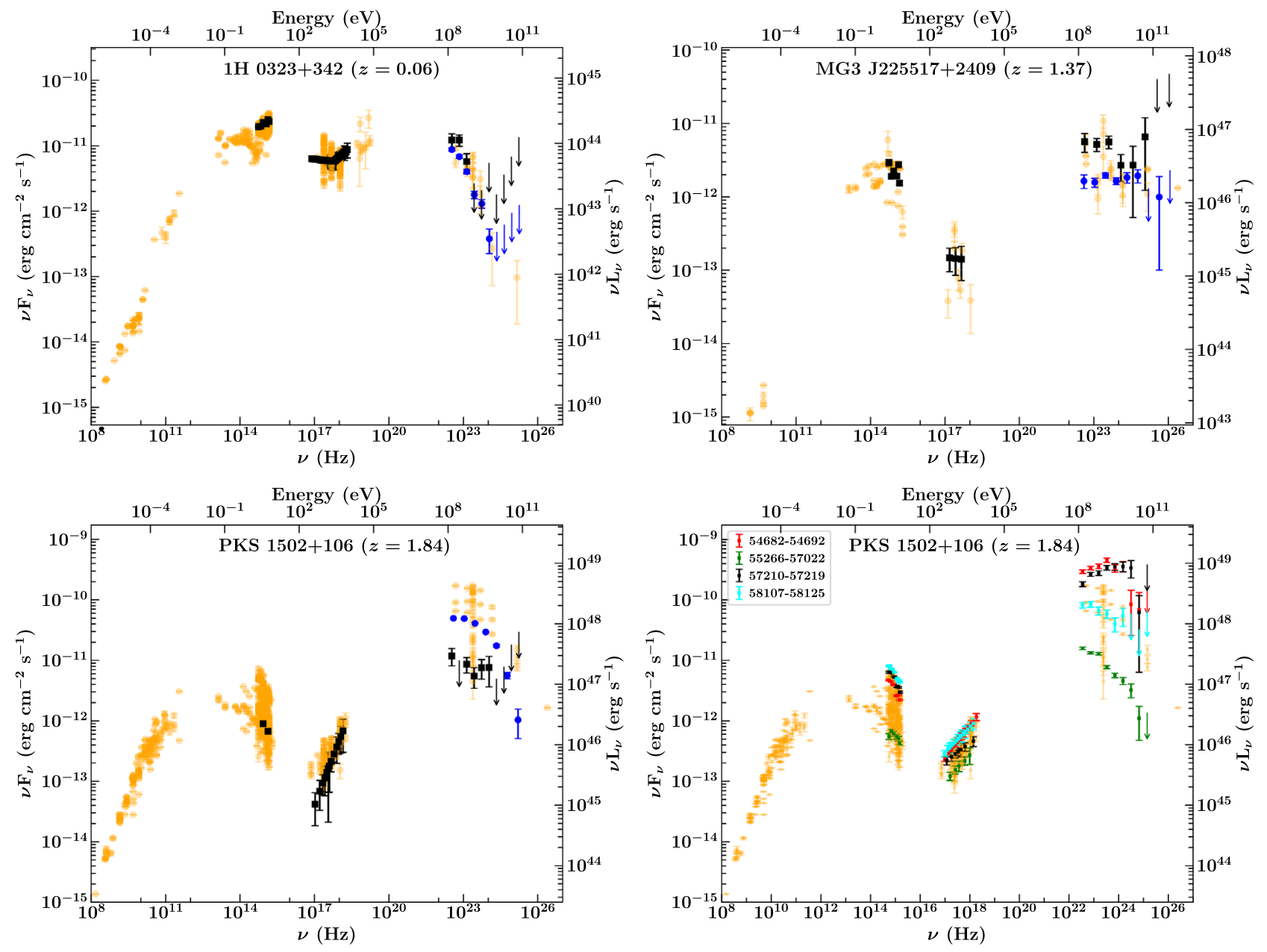

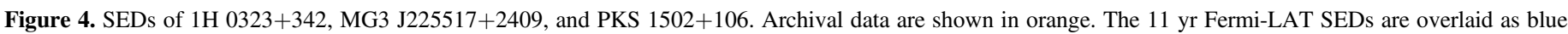

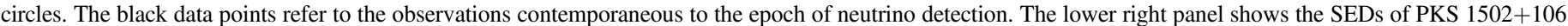
during four selected epochs (see Table 5 for details).

(IceCube Collaboration 2019a). It is the 15th brightest out of 2863 sources in the 4LAC catalog in terms of gamma-ray energy flux at $>100 \mathrm{MeV}$ despite its large redshift of 1.84 (Hewett \& Wild 2010), suggesting an extremely high intrinsic luminosity.

It was found to be in a low-activity state during the arrival time of the high-energy neutrino (see Figures 4 and A5). However, the OVRO radio light curve of PKS $1502+106$ shows a long-term outburst starting in 2014 and reaching the highest flux density ever reported from this source (since the beginning of the OVRO measurements in 2008) during the arrival of the $300 \mathrm{TeV}$ neutrino IC-190730A (Kiehlmann et al. 2019). The object TXS 0506+056 showed a similar increase in the radio emission observed by OVRO in coincidence with IC170922A (Aartsen et al. 2018a; Kiehlmann et al. 2019). A strong increase in radio emission was also determined in very long baseline interferometry (VLBI) data for another blazar, PKS B1424-418, which was found coincident with a highenergy but poorly reconstructed neutrino event (Kadler et al. 2016). Plavin et al. (2020) found a correlation of IceCube neutrinos with radio-bright AGNs with a $0.2 \% p$-value. Quantifying the chance coincidence of a radio flare with the arrival time of a neutrino is outside the scope of this paper.

Figure 4 (lower left panel) shows the broadband SED of PKS $1502+106$. The $11 \mathrm{yr}$ averaged gamma-ray spectrum of this source reveals a significant curvature/break that could be reflecting the shape of the particle spectrum or due to extrinsic absorption by the BLR photons. Interestingly, the EBL absorption is not significant below $50 \mathrm{GeV}$ at $z=1.84$ (Fermi-LAT Collaboration et al. 2018), and the gamma-ray emission from PKS $1502+106$ has been explained by the interaction of the jet electrons with the BLR photons (e.g., Abdo et al. 2010). Therefore, the observed spectral curvature could be due to gamma-ray absorption by the BLR photons via the pair-production process and/or a transition from the Thomson to Klein-Nishina regime. If so, the same BLR photon field could also act as a target photon field for neutrino production by interacting with the hadrons present in the jet (see, e.g., Rodrigues et al. 2019).

The gamma-ray spectral index shows a variation in time (see Figure A5, second panel). The spectrum tends to harden when the gamma-ray flux increases. The hard spectral regions indicate an increase in high-energy emission and are therefore promising targets for follow-up searches of $\mathcal{O}(\mathrm{TeV})$ neutrinos. Since PKS $1502+106$ is the most interesting source of our sample (due to its high gamma-ray energy flux), we study the spectral behavior during the multiwavelength flares in more detail to give guidance for future neutrino searches. We split the $11 \mathrm{yr}$ light curve into four regions of interest, where we obtain the gamma-ray spectral shape (see Table 5). We select 
Table 5

Summary of SED Analysis

\begin{tabular}{|c|c|c|c|c|c|c|}
\hline \multicolumn{7}{|c|}{ Fermi-LAT } \\
\hline \multirow[t]{2}{*}{ Name } & \multirow{2}{*}{$\begin{array}{c}\text { Time Window } \\
\text { MJD }\end{array}$} & \multirow{2}{*}{$\begin{array}{c}\text { Flux } \\
10^{-7} \mathrm{ph} \mathrm{cm}^{-2} \mathrm{~s}^{-1}\end{array}$} & \multirow[t]{2}{*}{ Power-law Index } & \multicolumn{2}{|c|}{ log-parabola Indices } & \multirow[t]{2}{*}{ TS } \\
\hline & & & & $\alpha$ & $\beta$ & \\
\hline \multicolumn{7}{|c|}{$11 \mathrm{yr}$ Averaged } \\
\hline $1 \mathrm{H} 0323+342$ & $54,682-58,633$ & $0.45 \pm 0.02$ & $\cdots$ & $2.77 \pm 0.04$ & $0.09 \pm 0.04$ & 1027 \\
\hline PKS $1502+106$ & $54,682-58,695$ & $2.97 \pm 0.02$ & $\ldots$ & $2.12 \pm 0.01$ & $0.10 \pm 0.01$ & 95412 \\
\hline MG3 J225517+2409 & $54,682-58,633$ & $0.11 \pm 0.01$ & $2.03 \pm 0.04$ & $\cdots$ & $\cdots$ & 955 \\
\hline \multicolumn{7}{|c|}{ Contemporaneous } \\
\hline $1 \mathrm{H} 0323+342$ & $57,263-57,392$ & $1.24 \pm 0.21$ & $\ldots$ & $3.25 \pm 0.47$ & $0.24 \pm 0.25$ & 90 \\
\hline PKS $1502+106$ & $58,664-58,724$ & $0.86 \pm 0.22$ & $\cdots$ & $2.31 \pm 0.16$ & $0.01 \pm 0.07$ & 97 \\
\hline MG3 J225517+2409 & $55,346-55,501$ & $0.41 \pm 0.08$ & $2.02 \pm 0.09$ & $\cdots$ & $\cdots$ & 181 \\
\hline \multicolumn{7}{|c|}{ Interesting Multiwavelength Features of PKS $1502+106$} \\
\hline PKS $1502+106$ & $54,682-54,692$ & $19.48 \pm 0.81$ & .. & $1.87 \pm 0.04$ & $0.12 \pm 0.02$ & 4205 \\
\hline & $55,266-57,022$ & $0.88 \pm 0.03$ & $\ldots$ & $2.27 \pm 0.02$ & $0.07 \pm 0.01$ & 56318 \\
\hline & $57,210-57,219$ & $14.15 \pm 0.69$ & $\ldots$ & $1.74 \pm 0.05$ & $0.09 \pm 0.02$ & 4830 \\
\hline & $58,107-58,125$ & $4.80 \pm 0.35$ & $\cdots$ & $2.16 \pm 0.06$ & $0.06 \pm 0.04$ & 25676 \\
\hline \multicolumn{7}{|c|}{ Swift-XRT } \\
\hline Name & $\begin{array}{c}\text { Exposure } \\
\text { ks }\end{array}$ & $\Gamma_{1}$ & $\Gamma_{2}$ & $\begin{array}{c}\text { Flux } \\
10^{-12} \mathrm{erg} \mathrm{cm}^{-2} \mathrm{~s}^{-1}\end{array}$ & $\begin{array}{c}\text { Normalization } \\
10^{-4} \mathrm{ph} \mathrm{cm}^{-2} \mathrm{~s}^{-1} \mathrm{keV}^{-1}\end{array}$ & $\chi^{2} /$ dof \\
\hline \multicolumn{7}{|c|}{ Contemporaneous } \\
\hline $1 \mathrm{H} 0323+342$ & 32.81 & $2.04_{-0.04}^{+0.04}$ & $1.67_{-0.13}^{+0.10}$ & $22.94_{-0.64}^{+0.57}$ & $37.50_{-0.69}^{+0.70}$ & $366.91 / 285$ \\
\hline PKS $1502+106$ & 5.61 & $1.00_{-0.31}^{+0.30}$ & & $1.12_{-0.28}^{+0.41}$ & $0.67_{-0.18}^{+0.21}$ & $56.59 / 66$ \\
\hline MG3 J225517+2409 & 1.65 & $2.03_{-1.06}^{+1.00}$ & & $0.50_{-0.20}^{+0.53}$ & $0.90_{-0.34}^{+0.45}$ & $12.57 / 12$ \\
\hline \multicolumn{7}{|c|}{ Interesting Multiwavelength Features of PKS $1502+106$} \\
\hline \multirow[t]{4}{*}{ PKS $1502+106$} & 36.67 & $1.44_{-0.06}^{+0.06}$ & & $2.16_{-0.12}^{+0.14}$ & $2.44_{-0.13}^{+0.12}$ & $63.62 / 69$ \\
\hline & 13.50 & $1.58_{-0.25}^{+0.25}$ & & $0.73_{-0.13}^{+0.17}$ & $0.94_{-0.15}^{+0.14}$ & $12.51 / 7$ \\
\hline & 11.06 & $1.69_{-0.16}^{+0.17}$ & & $1.22_{-0.15}^{+0.17}$ & $1.75_{-0.21}^{+0.21}$ & $20.60 / 12$ \\
\hline & 14.69 & $1.57_{-0.10}^{+0.10}$ & & $2.12_{-0.20}^{+0.21}$ & $2.72_{-0.21}^{+0.21}$ & $42.60 / 29$ \\
\hline & & & Swift-UVOT & & & \\
\hline Name & $V$ & $B$ & $U$ & $W 1$ & $M 2$ & $W 2$ \\
\hline \multicolumn{7}{|c|}{ Contemporaneous } \\
\hline $1 \mathrm{H} 0323+342$ & $19.58 \pm 0.23$ & $19.85 \pm 0.19$ & $22.82 \pm 0.26$ & $21.58 \pm 0.30$ & $24.94 \pm 0.38$ & $23.47 \pm 0.31$ \\
\hline PKS $1502+106$ & & & $0.90 \pm 0.06$ & & $0.67 \pm 0.06$ & \\
\hline MG3 J225517+2409 & $2.91 \pm 0.32$ & $1.90 \pm 0.13$ & $2.26 \pm 0.12$ & $1.92 \pm 0.12$ & $2.74 \pm 0.25$ & $1.54 \pm 0.10$ \\
\hline \multicolumn{7}{|c|}{ Interesting Multiwavelength Features of PKS $1502+106$} \\
\hline PKS $1502+106$ & $4.71 \pm 0.11$ & $4.45 \pm 0.08$ & $3.97 \pm 0.08$ & $2.59 \pm 0.06$ & $2.67 \pm 0.07$ & $2.21 \pm 0.06$ \\
\hline & $0.55 \pm 0.06$ & $0.67 \pm 0.05$ & $0.61 \pm 0.06$ & $0.55 \pm 0.05$ & $0.51 \pm 0.04$ & $0.43 \pm 0.03$ \\
\hline & $6.38 \pm 0.24$ & $6.24 \pm 0.18$ & $5.32 \pm 0.16$ & $3.71 \pm 0.14$ & $3.57 \pm 0.11$ & $2.98 \pm 0.11$ \\
\hline & $8.06 \pm 0.20$ & $7.20 \pm 0.15$ & $6.42 \pm 0.15$ & $5.03 \pm 0.16$ & $4.68 \pm 0.13$ & $4.34 \pm 0.10$ \\
\hline
\end{tabular}

Note. The first block shows the Fermi-LAT SED results performed in a given time window. The quoted gamma-ray flux is integrated in the $0.1-800 \mathrm{GeV}$ energy range. The SED is modeled with a power law, unless a log-parabola description with spectral parameters $\alpha$ and $\beta$ results in a significantly better fit to the data. The second block shows the results of the Swift-XRT spectral analysis. Here $\Gamma_{1}$ is the photon index of a power-law model or photon index before the break energy in a broken power-law model, while $\Gamma_{2}$ is the photon index after the break energy in a broken power-law model. The flux is integrated in the $0.3-10 \mathrm{keV}$ energy range, and the normalization is defined at $1 \mathrm{keV}$ in units of $10^{-4} \mathrm{ph} \mathrm{cm}^{-2} \mathrm{~s}^{-1} \mathrm{keV}^{-1}$. Absorption by Galactic neutral hydrogen is taken into account using the following column densities along the line of sight (Kalberla et al. 2005): $N_{\mathrm{H}}=1.17 \times 10^{21}(1 \mathrm{H} 0323+342), 2.03 \times 10^{20}(\mathrm{PKS} 1502+106)$, and $3.57 \times 10^{20} \mathrm{~cm}^{-2}$ (MG3 J225517+2409). The third block shows the results of the Swift-UVOT analysis and gives the average flux in the Swift $V, B, U, W 1, M 2$, and $W 2$ bands in units of $10^{-12} \mathrm{erg} \mathrm{cm}^{-2} \mathrm{~s}^{-1}$. The Swift analyses were performed in the same time periods specified for the Fermi-LAT results.

one period from MJD 55,266-57,022 to cover the quiet state in gamma-rays and three short periods of roughly 10 days in length chosen to cover the three bright X-ray flares, which are also accompanied by optical flares (see Figure A5). We find that during the gamma-ray quiet state, the flux values in each wavelength reach a minimum flux level (shown in green). Interestingly, the highest flare in the X-ray and optical (cyan) does not correspond to the highest flare in gamma-rays, while 

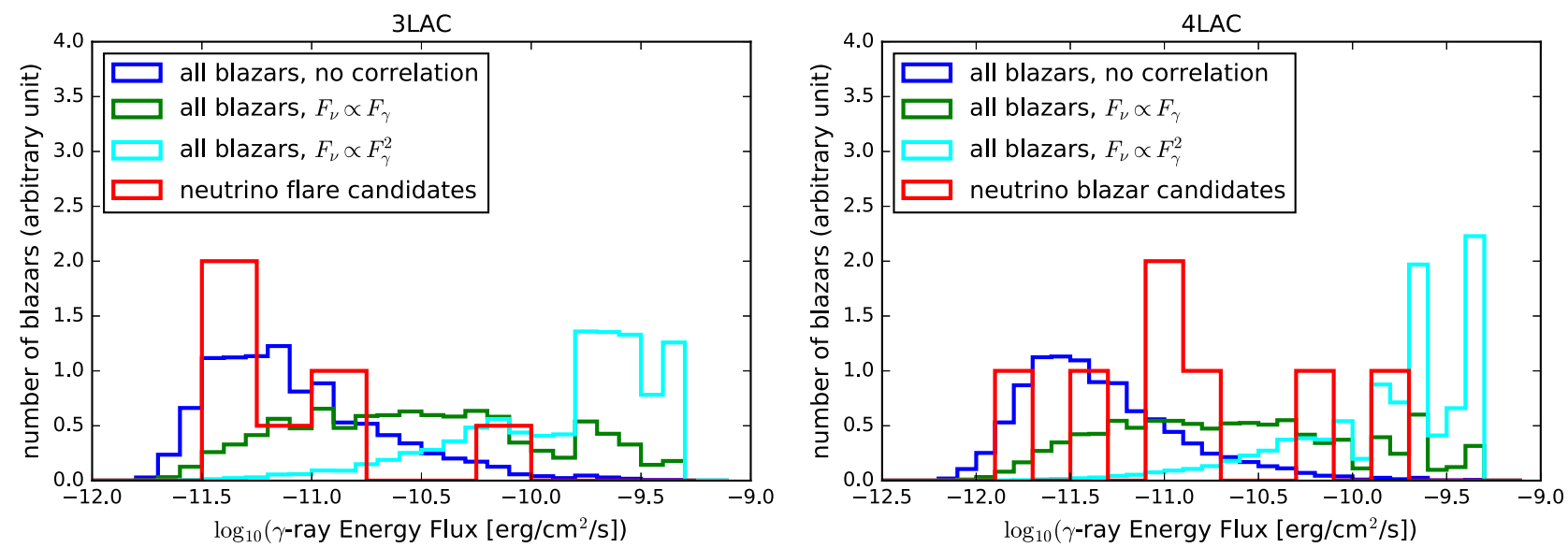

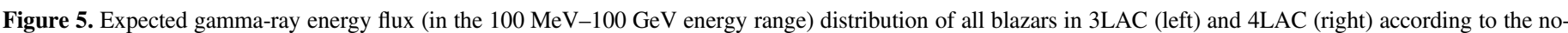

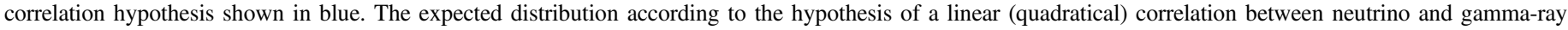

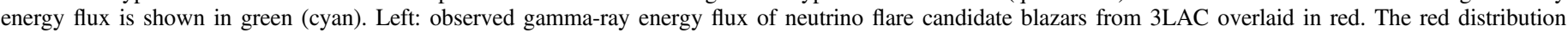

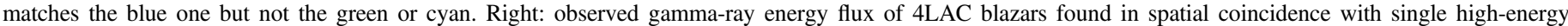
neutrinos (red).

the highest gamma-ray activity is also accompanied by a significant increase in the optical and X-ray. The different flaring behavior indicates different conditions of the emission region in the source. Detailed time-dependent modeling, which is outside the scope of this work, could give deeper insight into the variable nature of the source.

\subsection{Source Population}

As a result of the K-S test (see Table 4), we find that the blazars found in coincidence with single high-energy neutrinos are well described by both the gamma-ray energy flux distribution expected in the case of a linear correlation between neutrino and gamma-ray energy flux and the hypothesis of no correlation between the two fluxes. If all sources are combined, the single neutrino source candidates are compatible with the no-correlation hypothesis with a $p$-value of $12.6 \%$ and consistent with the expectation in the case of a linear correlation between neutrino and gamma-ray energy flux ( $p$-value of $64 \%$ ). They show a mismatch with the hypothesis of a quadratical correlation ( $p$-value of $0.03 \%$ ). The gamma-ray energy flux distribution is illustrated in Figure 5.

The object MG3 J225517+2409 would have failed our angular uncertainty requirement selection and was not included in the K-S test.

At the same time, the candidate neutrino flare sources show a good match with the random distribution ( $p$-value of 39\%$98 \%)$ but are less well described by the energy flux-weighted distribution ( $p$-value of $0.4 \%-2.1 \%$ ).

\section{Summary and Conclusions}

\subsection{Individual Sources}

In summary, the available data do not show any significant temporal correlation with the neutrino arrival time considering all blazars studied here, which would allow us to identify one of the sources as a potential cosmic-ray acceleration site. This is consistent with findings by Righi et al. (2019), who studied the gamma-ray light curves of seven BL Lac objects and did not find a clear pattern in common among the sources.

Most sources are well observed in GeV gamma-rays and optical wavelengths, where no significant temporal correlation with the neutrino emission is found. For the sources monitored by OVRO, no short-term features related to the neutrino arrival time are observed. Three out of five sources coincident with single high-energy neutrinos are monitored by OVRO in radio, and two (TXS 0506+056 and PKS 1502+106) show a longterm increase of the radio flux density, which peaks during the neutrino arrival time. The third one (MG3 J225517+2409) is only covered by OVRO observations 70 days after the neutrino arrival time but might be compatible with a radio flux increase assuming a smooth extrapolation of the temporal behavior to earlier times. The five neutrino flare source candidates, which are monitored in radio, show no correlation with long-term radio activity. Radio monitoring of future neutrino blazar coincidences could reveal whether there is indeed a connection between the radio and single high-energy neutrino emission.

\subsection{Source Population}

We find that the single high-energy neutrino coincidences with blazars are consistent with a $p$-value of $12.6 \%$ with being due to random chance. At the same time, they are consistent with the hypothesis that the single high-energy neutrino emission is correlated linearly with the gamma-ray brightness of the blazars. This interpretation is consistent with the association of IC-170922A and the bright gamma-ray flare of TXS $0506+056$.

We note that the contribution of gamma-ray blazars to the diffuse neutrino flux is constrained by blazar stacking analyses (Aartsen et al. 2017d), which constrain the blazar contribution to less than $27 \%$ under the assumption that the neutrino spectrum follows an unbroken $E^{-2.5}$ power law. Assuming a steeper power law with an index of 2 , which is compatible with the diffuse flux fit above $\sim 200 \mathrm{TeV}$, weakens the constraints on the diffuse flux contribution to $40 \%-80 \%$ (Aartsen et al. 2018b) and leaves room for a significant contribution from gamma-ray blazars. Krauß et al. (2018) studied the gamma-ray and X-ray emission of 3LAC blazars in the vicinity of highenergy starting events detected by IceCube and found no direct correlation between the Fermi-LAT gamma-ray flux and the IceCube neutrino flux.

Assuming that the observed linear correlation of single highenergy neutrinos and the average gamma-ray energy flux is 
genuine, our results would have many broad implications. They would allow us to utilize neutrino blazar coincidences for the study of cosmic-ray acceleration. Furthermore, they would allow for an effective search for more coincidences to further characterize the population of sources of high-energy neutrinos.

At the same time, the candidate neutrino flare-emitting blazars are compatible with the background hypothesis, and the data do not support the hypothesis that neutrino emission is correlated to the average gamma-ray energy flux. This could indicate that either neutrino flares are not accompanied by strong gamma-ray emission or these coincidences are of a random nature. The first case could be realized in sources where neutrinos are produced in regions optically thick to gamma-rays, where gamma-ray emission is absorbed (so-called hidden sources) and cascades to the X-ray band, where we do not have good observational constraints from archival data (see also Keivani et al. 2018; Gao et al. 2019).

This work was supported by the Initiative and Networking Fund of the Helmholtz Association. We thank Xavier Rodrigues, Shan Gao, and Walter Winter for fruitful discussions. B.J.S. is supported by NSF grants AST-1908952, AST1920392, and AST1911074. S.K. acknowledges support from the European Research Council under the European Union's Horizon 2020 research and innovation program, under grant agreement No.771282. W.M. acknowledges support from CONICYT project Basal AFB-170002. T.H. was supported by Academy of Finland projects 317383 and 320085.

Fermi-LAT: The Fermi-LAT Collaboration acknowledges generous ongoing support from a number of agencies and institutes that have supported both the development and the operation of the LAT, as well as scientific data analysis. These include the National Aeronautics and Space Administration and the Department of Energy in the United States; the Commissariat à l'Energie Atomique and the Centre National de la Recherche Scientifique/Institut National de Physique Nucléaire et de Physique des Particules in France; the Agenzia Spaziale Italiana and the Istituto Nazionale di Fisica Nucleare in Italy; the Ministry of Education, Culture, Sports, Science and Technology (MEXT), High Energy Accelerator Research Organization (KEK), and Japan Aerospace Exploration Agency
(JAXA) in Japan; and the K. A. Wallenberg Foundation, the Swedish Research Council, and the Swedish National Space Board in Sweden.

Additional support for science analysis during the operations phase is gratefully acknowledged from the Istituto Nazionale di Astrofisica in Italy and the Centre National d'Études Spatiales in France. This work was performed in part under DOE contract DE-AC02-76SF00515.

ASAS-SN: ASAS-SN is supported by the Gordon and Betty Moore Foundation through grant GBMF5490 to the Ohio State University and NSF grants AST-1515927 and AST-1908570. Development of ASAS-SN has been supported by NSF grant AST-0908816, the Mt. Cuba Astronomical Foundation, the Center for Cosmology and AstroParticle Physics at the Ohio State University, the Chinese Academy of Sciences South America Center for Astronomy (CAS-SACA), the Villum Foundation, and George Skestos.

Others: This research has made use of data from the OVRO $40 \mathrm{~m}$ monitoring program (Richards et al. 2011), which is supported in part by NASA grants NNX08AW31G, NNX11A043G, and NNX14AQ89G and NSF grants AST0808050 and AST-1109911.

The CSS survey is funded by the National Aeronautics and Space Administration under grant No. NNG05GF22G issued through the Science Mission Directorate Near-Earth Objects Observations Program. The CRTS survey is supported by the U.S. National Science Foundation under grants AST-0909182 and AST-1313422.

Part of this work is based on archival data, software, or online services provided by the Space Science Data Center (SSDC).

Software: Fermi-LAT ScienceTools (v11r04p00), fermipy (v0.17.4; Wood et al. 2017), astropy (Astropy Collaboration et al. 2013, 2018), IRAF (Tody 1986, 1993), ISIS (Alard \& Lupton 1998; Alard 2000), XSPEC (Arnaud 1996), HEASoft (v6.26.1).

\section{Appendix Multiwavelength Light-curve Plots}

Here, we present multiwavelength light curves (Figures A1-A14) for all sources in our sample. 

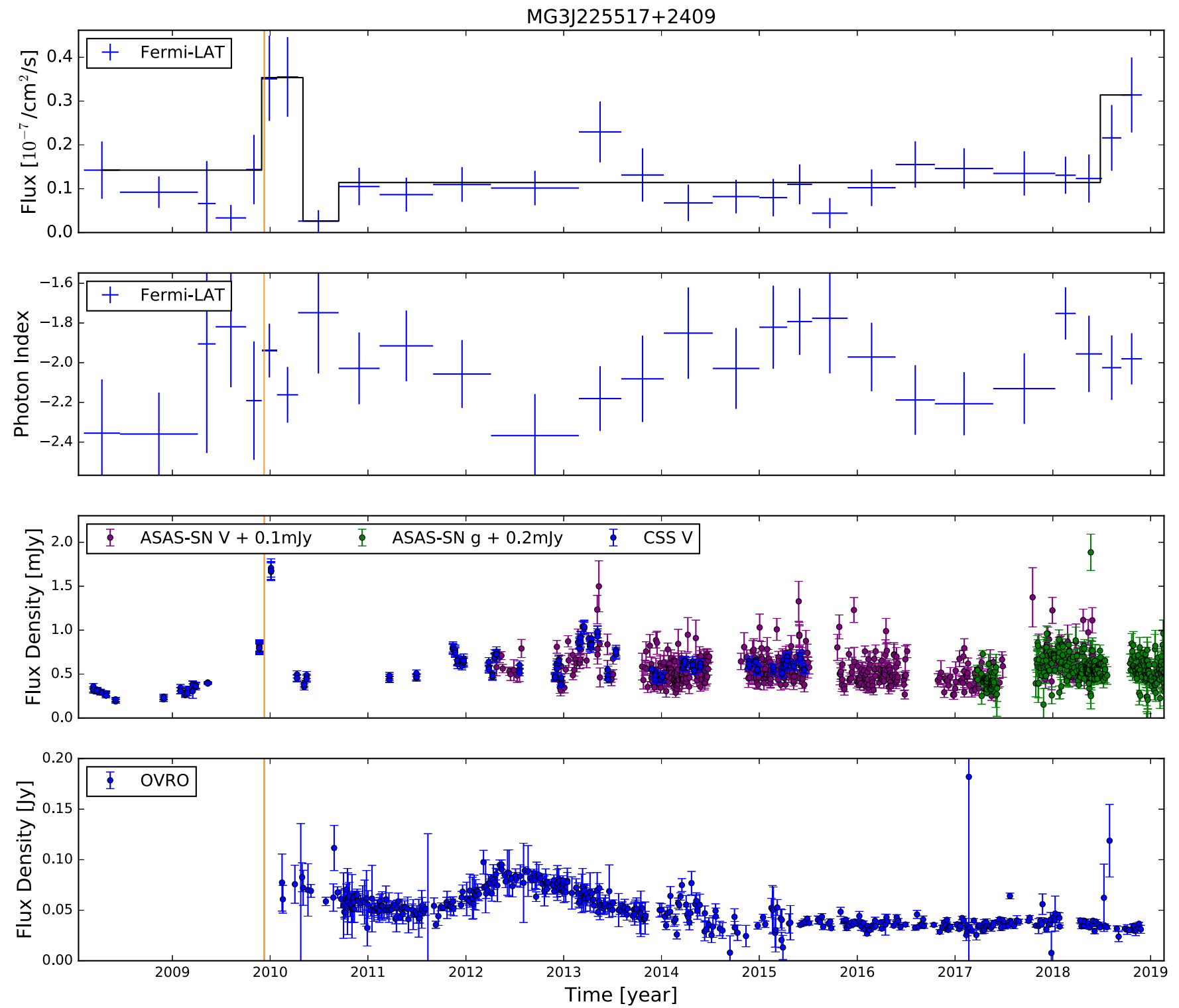

Figure A1. Multiwavelength light curve of MG3 J225517+2409. The orange line indicates the arrival time of the high-energy neutrino IC-100608A. 

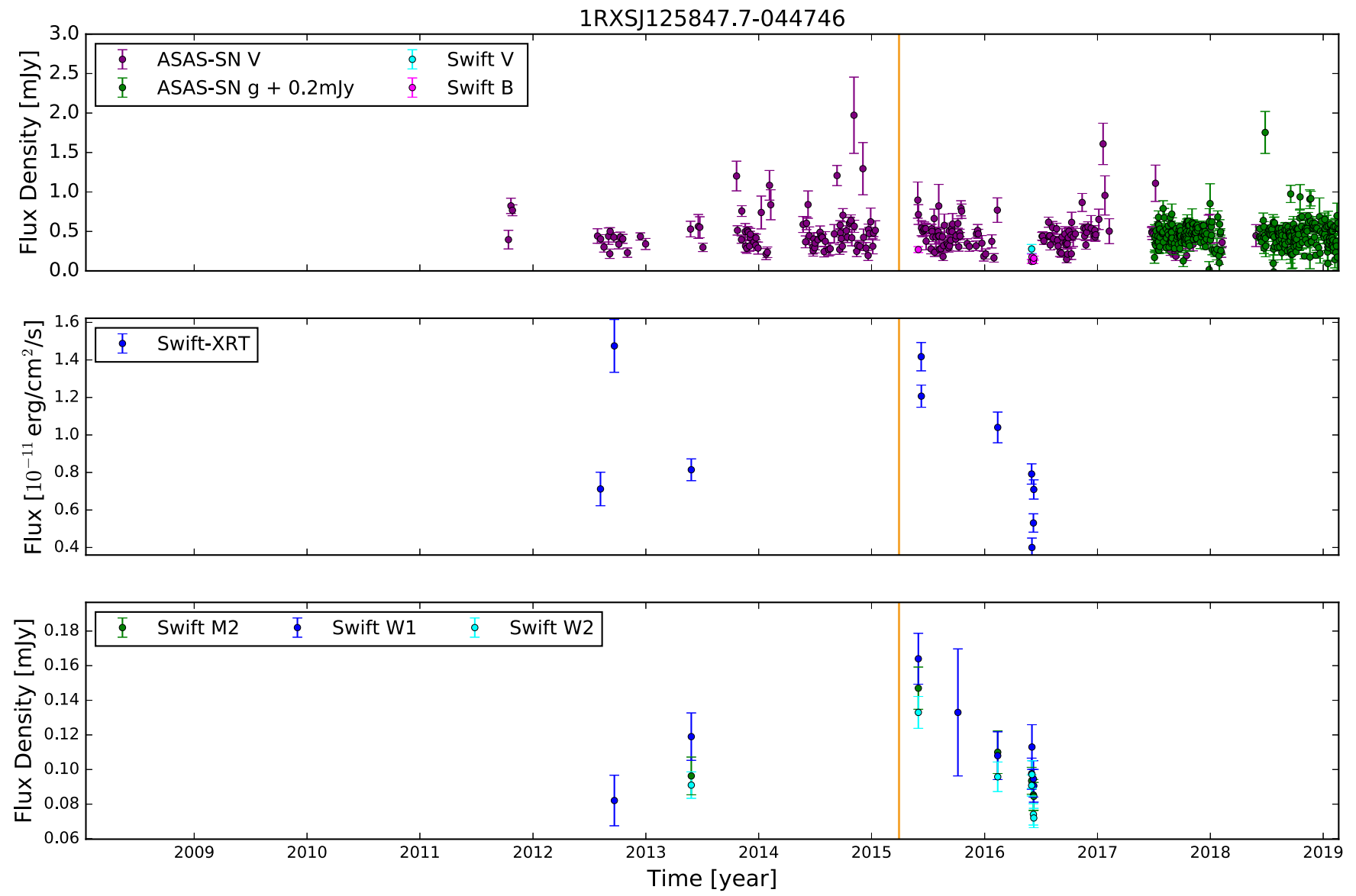

Figure A2. Multiwavelength light curve of 1RXS J125847.7-044746. The orange line indicates the arrival time of the high-energy neutrino IC-150926A. The source is too dim in gamma-rays to resolve the temporal variability.

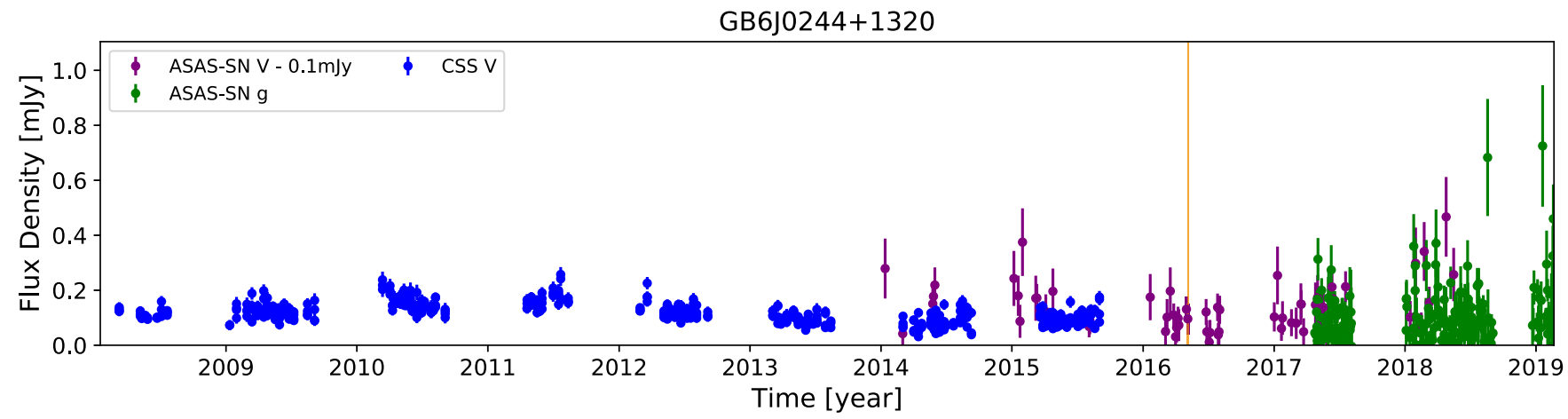

Figure A3. Multiwavelength light curve of GB6 J0244+1320. The orange line indicates the arrival time of the high-energy neutrino IC-161103A. The source is too dim in gamma-rays to resolve the temporal variability. 

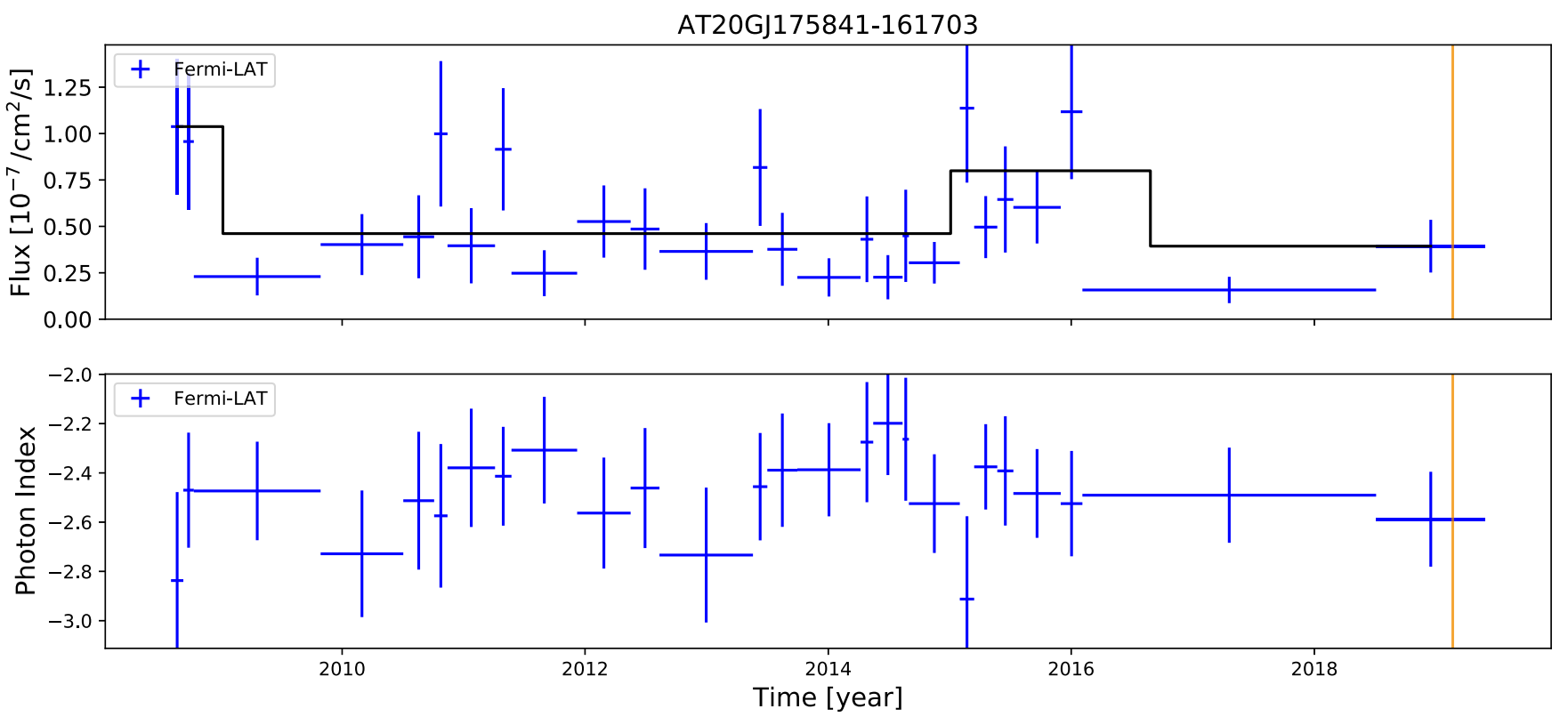

Figure A4. Multiwavelength light curve of AT20G J175841-161703. The orange line indicates the arrival time of the high-energy neutrino IC-190221A. 

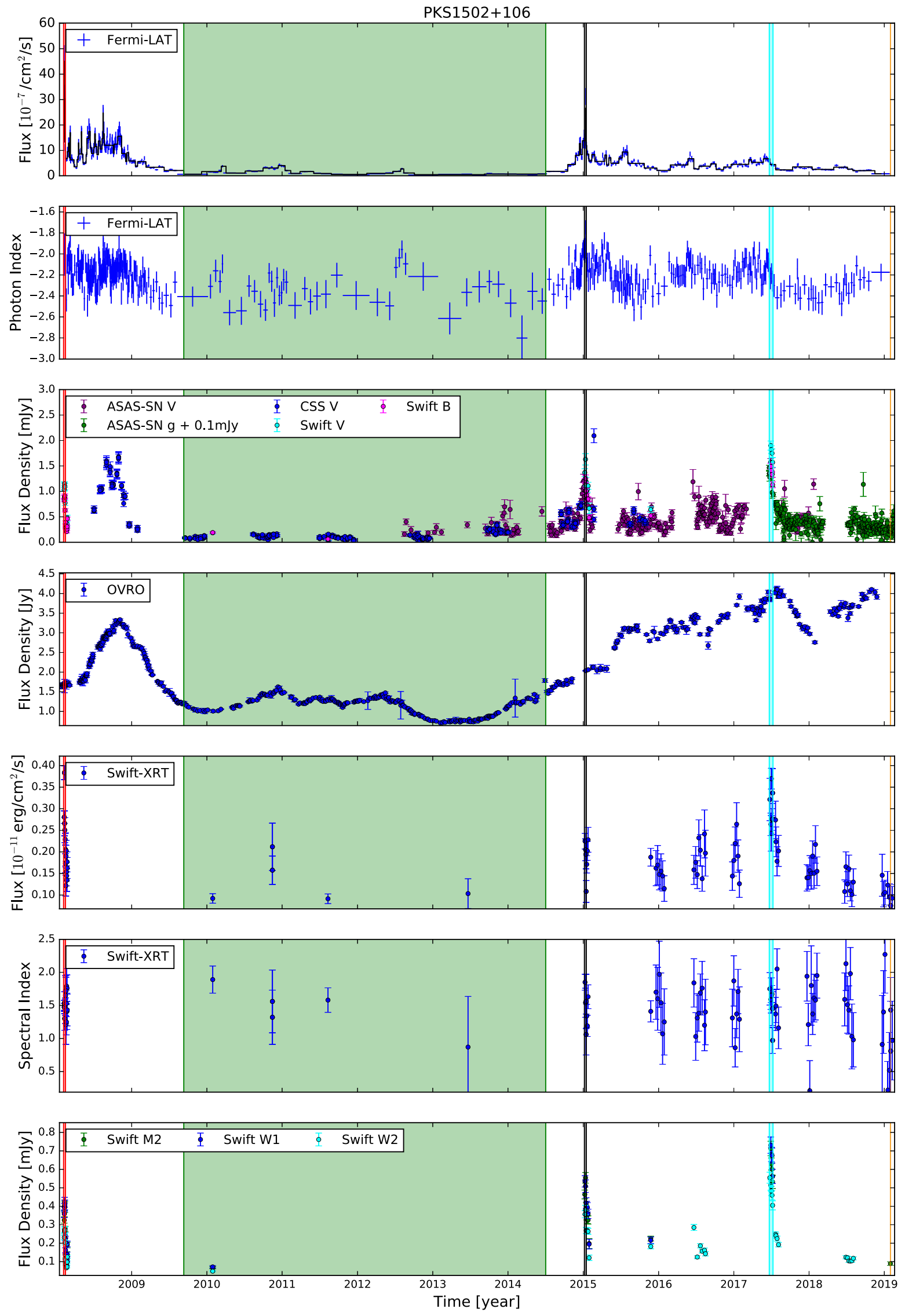

Figure A5. Multiwavelength light curve of PKS 1502+106. The orange line indicates the arrival time of the high-energy neutrino IC-190730A. The green region in the first panel marks the quiescent state, and vertical red, cyan, and black lines mark three flaring states, which are accompanied by X-ray and optical flares, selected for a dedicated gamma-ray spectral analysis (see Table 5 and Figure 4, which uses the same color code). 

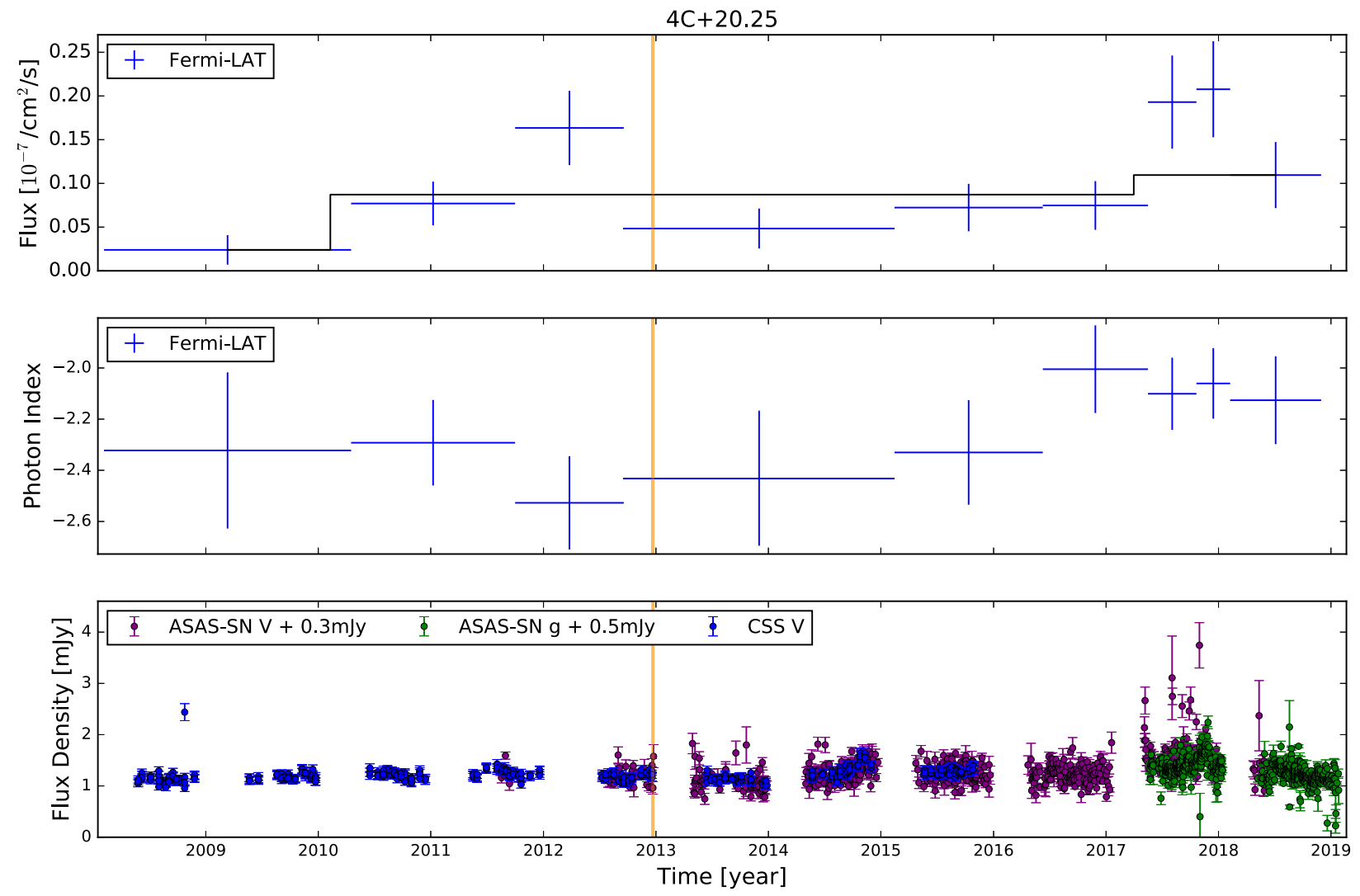

Figure A6. Multiwavelength light curve of $4 \mathrm{C}+20.25$. The duration of the neutrino flare is short $\left(T_{w}=5.2\right.$ days), and its arrival time is shown as an orange line.
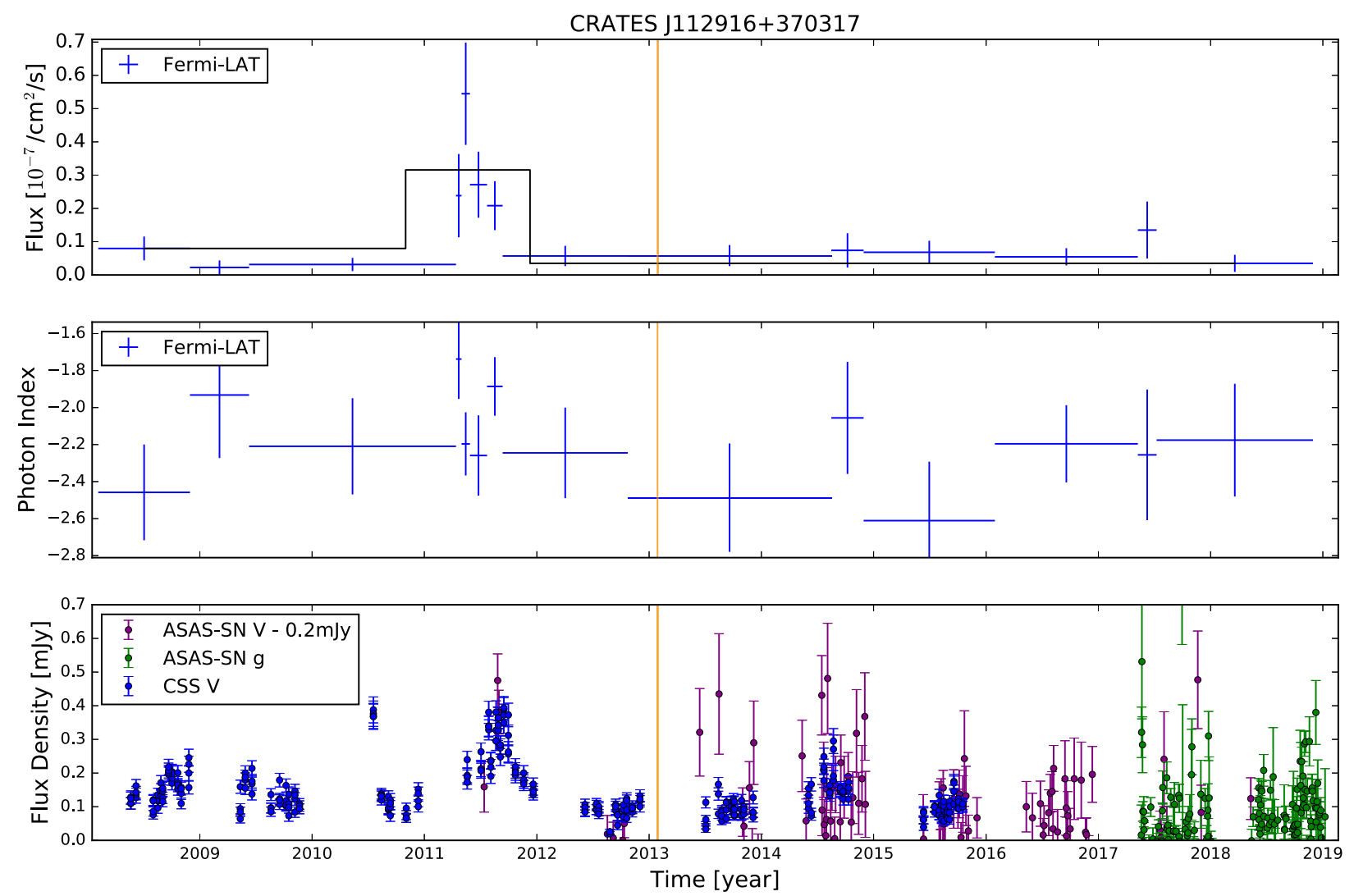

Figure A7. Multiwavelength light curve of CRATES J112916+370317. The duration of the neutrino flare is short $\left(T_{w}=1.4 \mathrm{hr}\right)$, and its arrival time is shown as an orange line. 

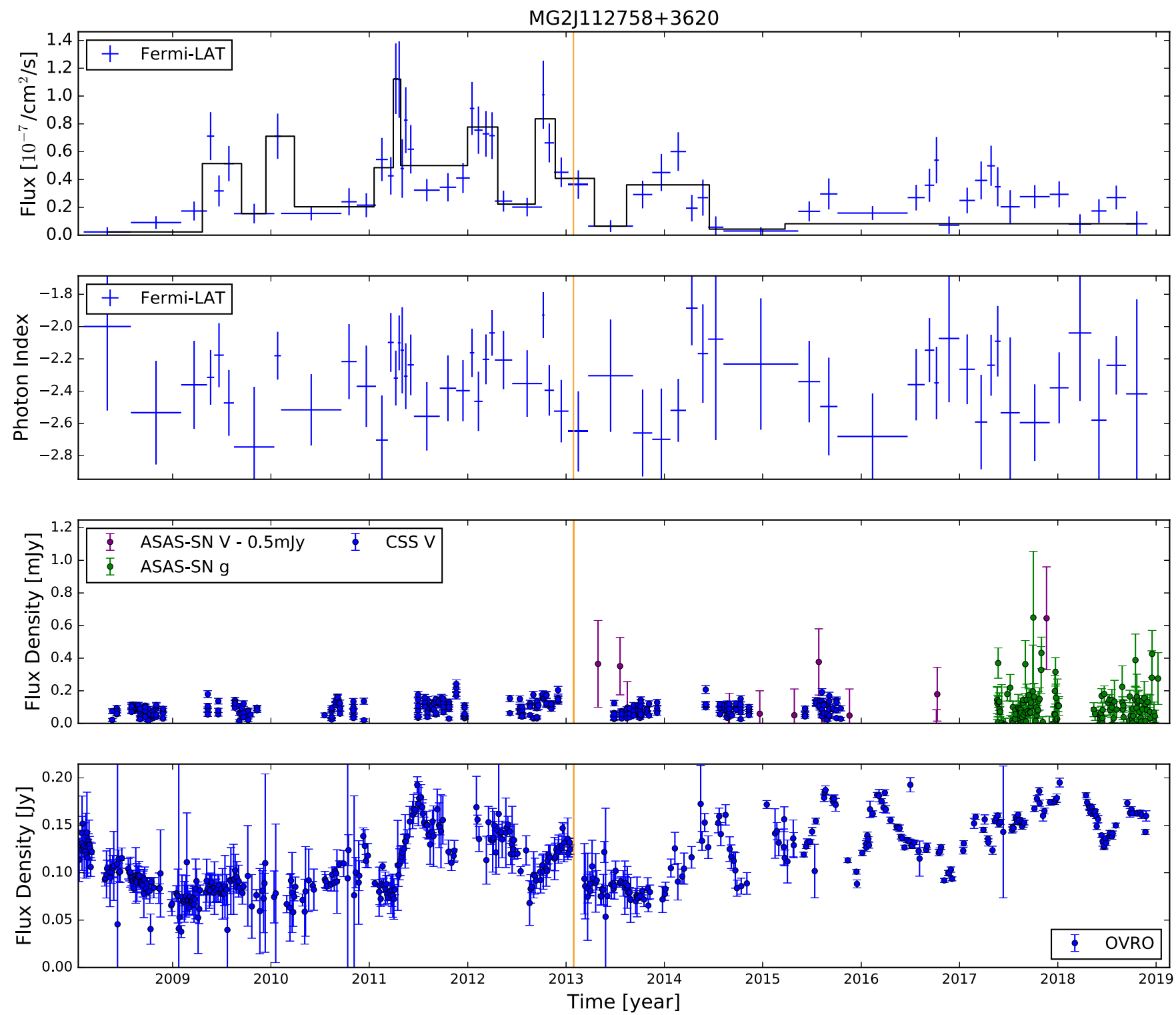

Figure A8. Multiwavelength light curve of MG2 J112758+3620. The duration of the neutrino flare is short $\left(T_{w}=1.4 \mathrm{hr}\right)$, and its arrival time is shown as an orange line. 

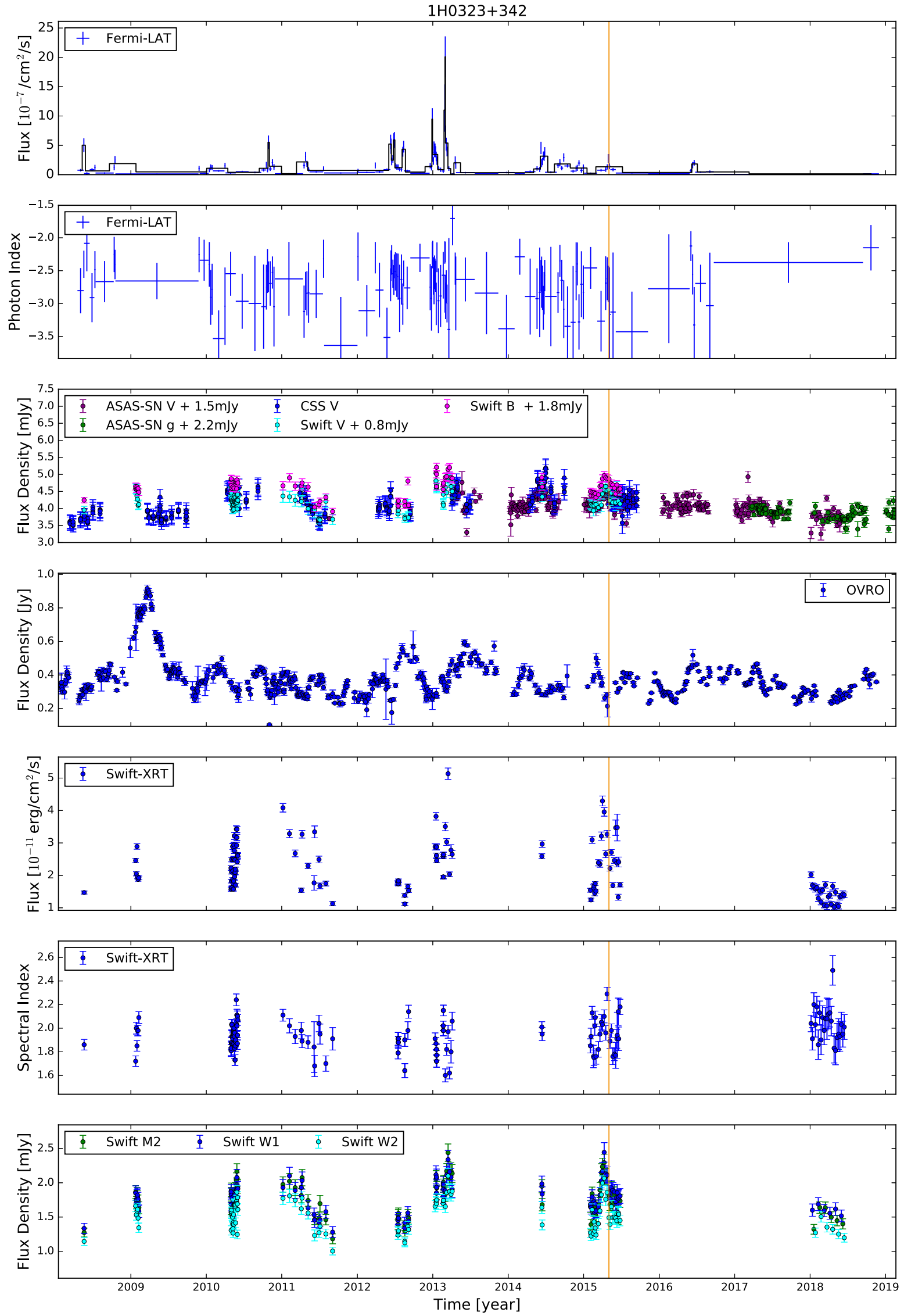

Figure A9. Multiwavelength light curve of $1 \mathrm{H} 0323+342$. The duration of the neutrino flare is short $\left(T_{w}=147 \mathrm{~s}\right)$, and its arrival time is shown as an orange line. 

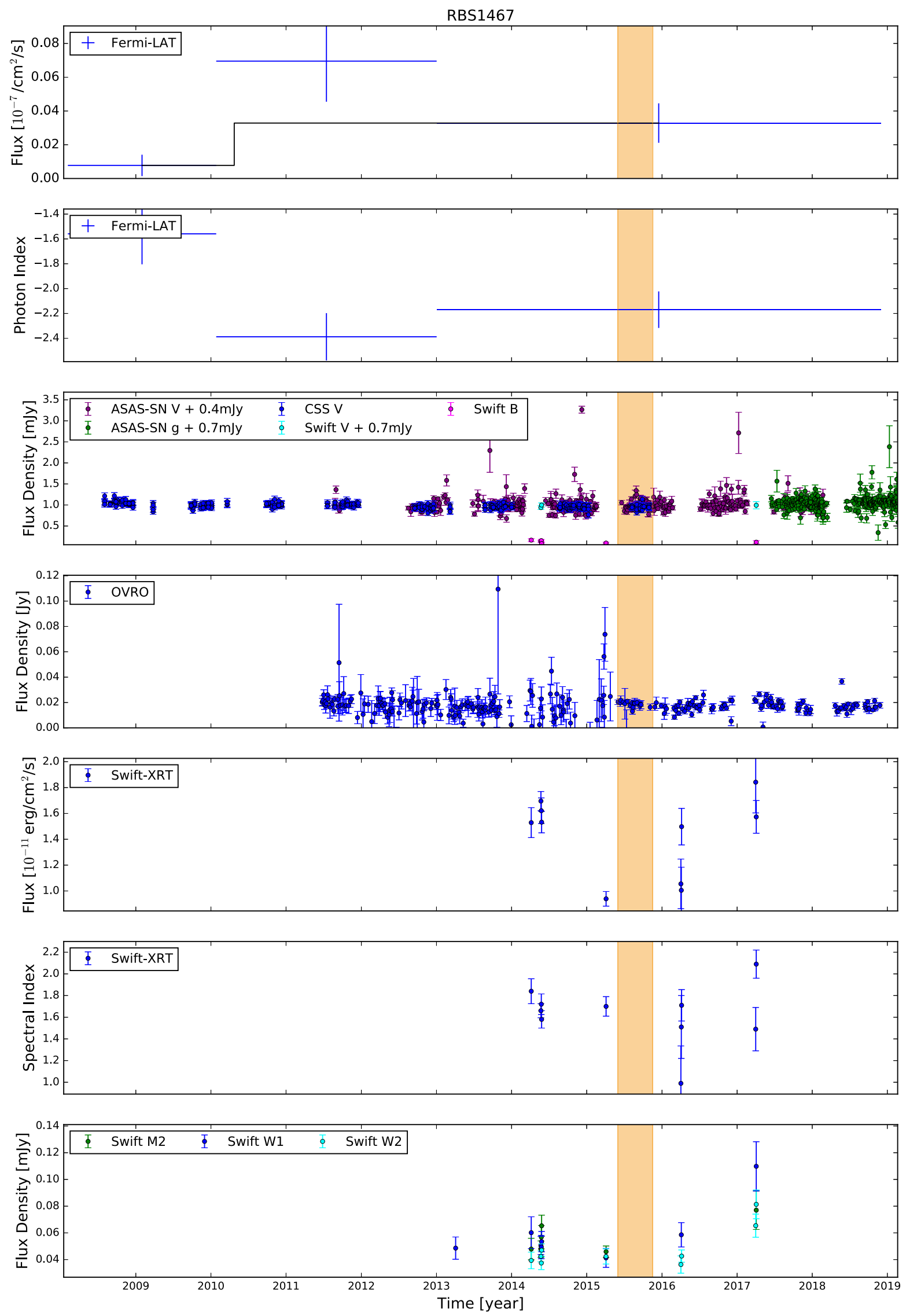

Figure A10. Multiwavelength light curve of RBS 1467. The orange shaded region is centered on the mean of the Gaussian describing the neutrino emission with a width corresponding to twice the standard deviation. 

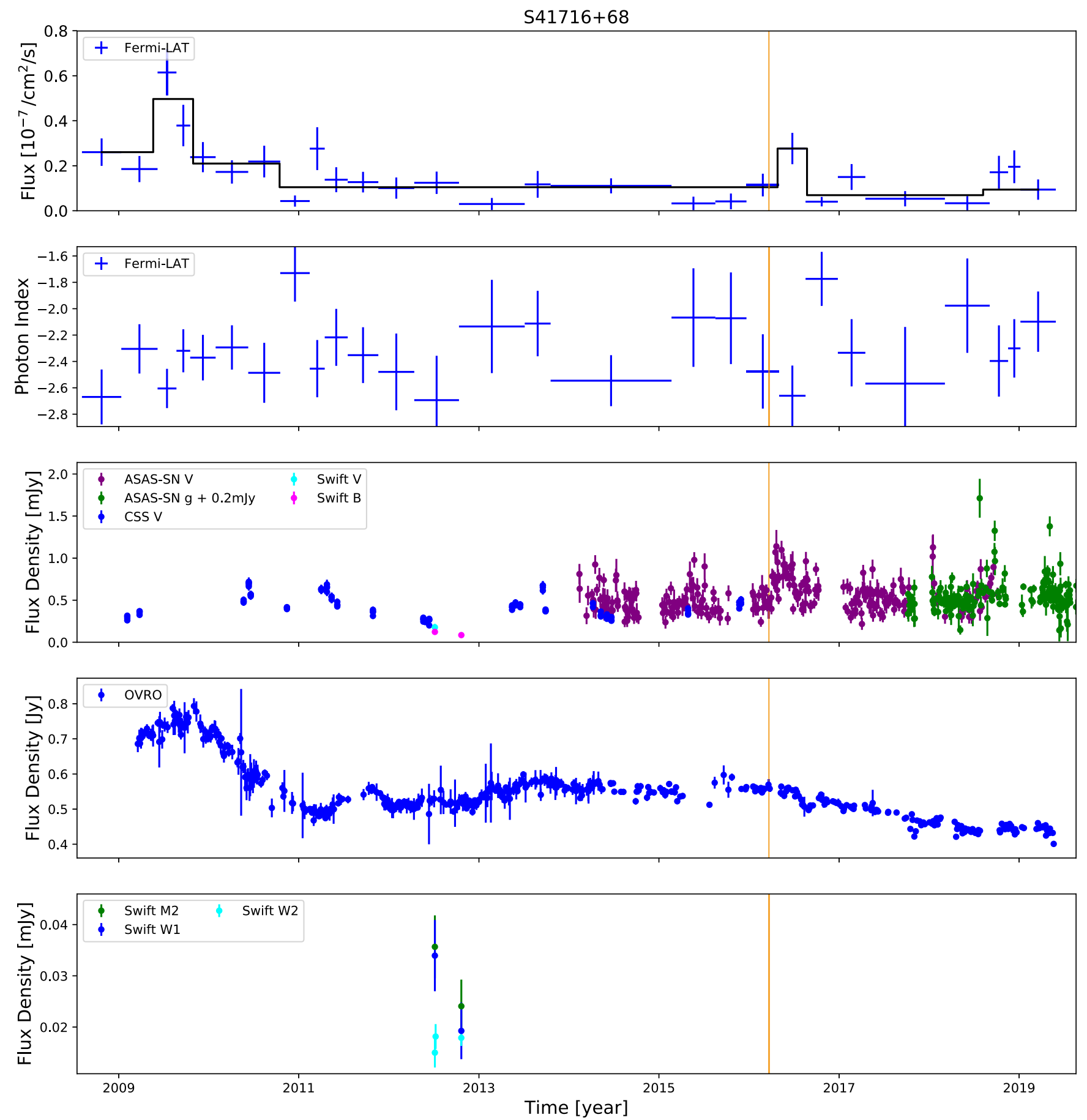

Figure A11. Multiwavelength light curve of S4 1716+68. The duration of the neutrino flare is short $\left(T_{w}=4.7 \mathrm{~s}\right)$, and its arrival time is shown as an orange line. 

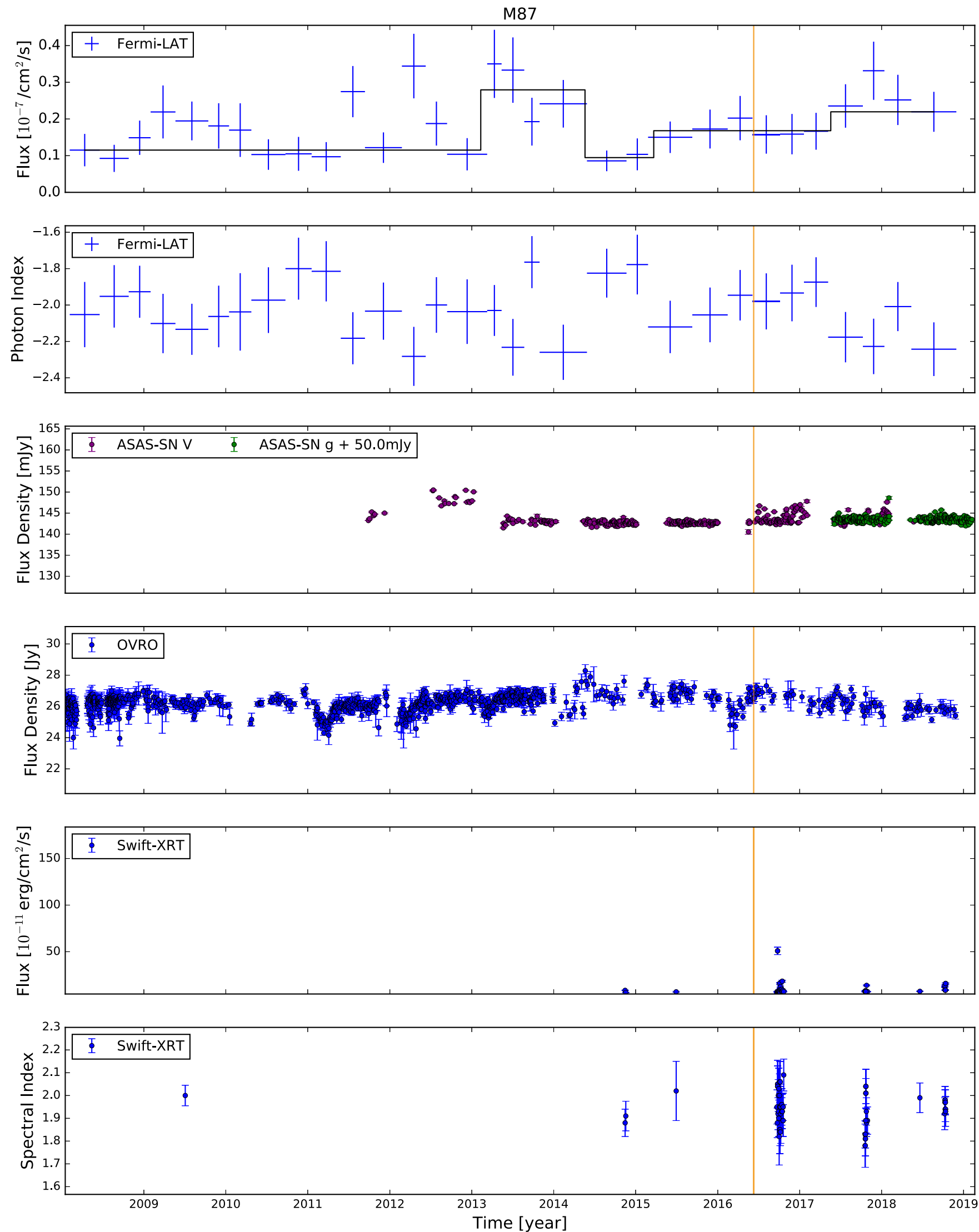

Figure A12. Multiwavelength light curve of M87. The duration of the neutrino flare is short ( $T_{w}=3.9$ minutes), and its arrival time is shown as an orange line. 

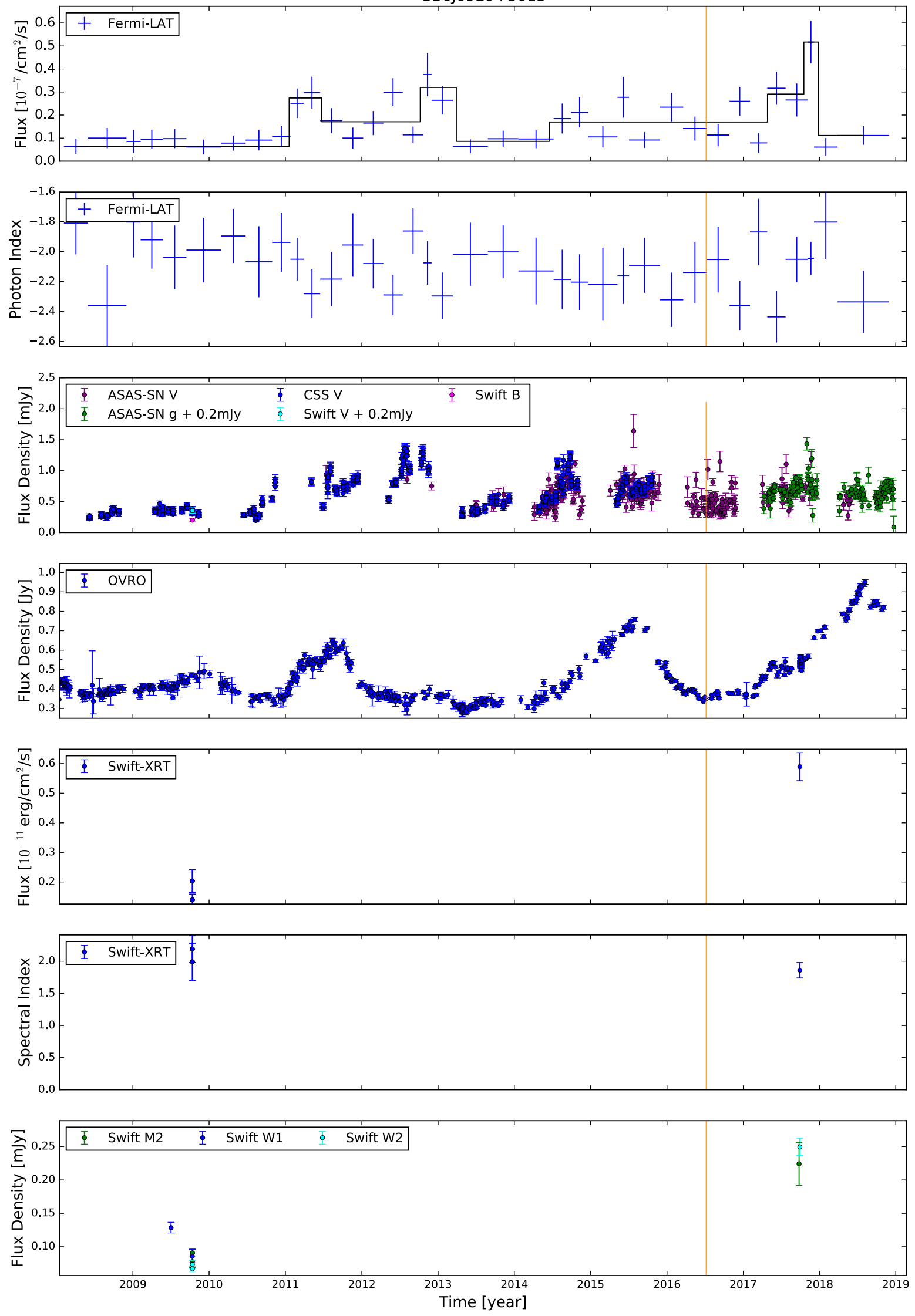

Figure A13. Multiwavelength light curve of GB6 J0929+5013. The duration of the neutrino flare is short ( $T_{w}=1.2$ days), and its arrival time is shown as an orange line. 

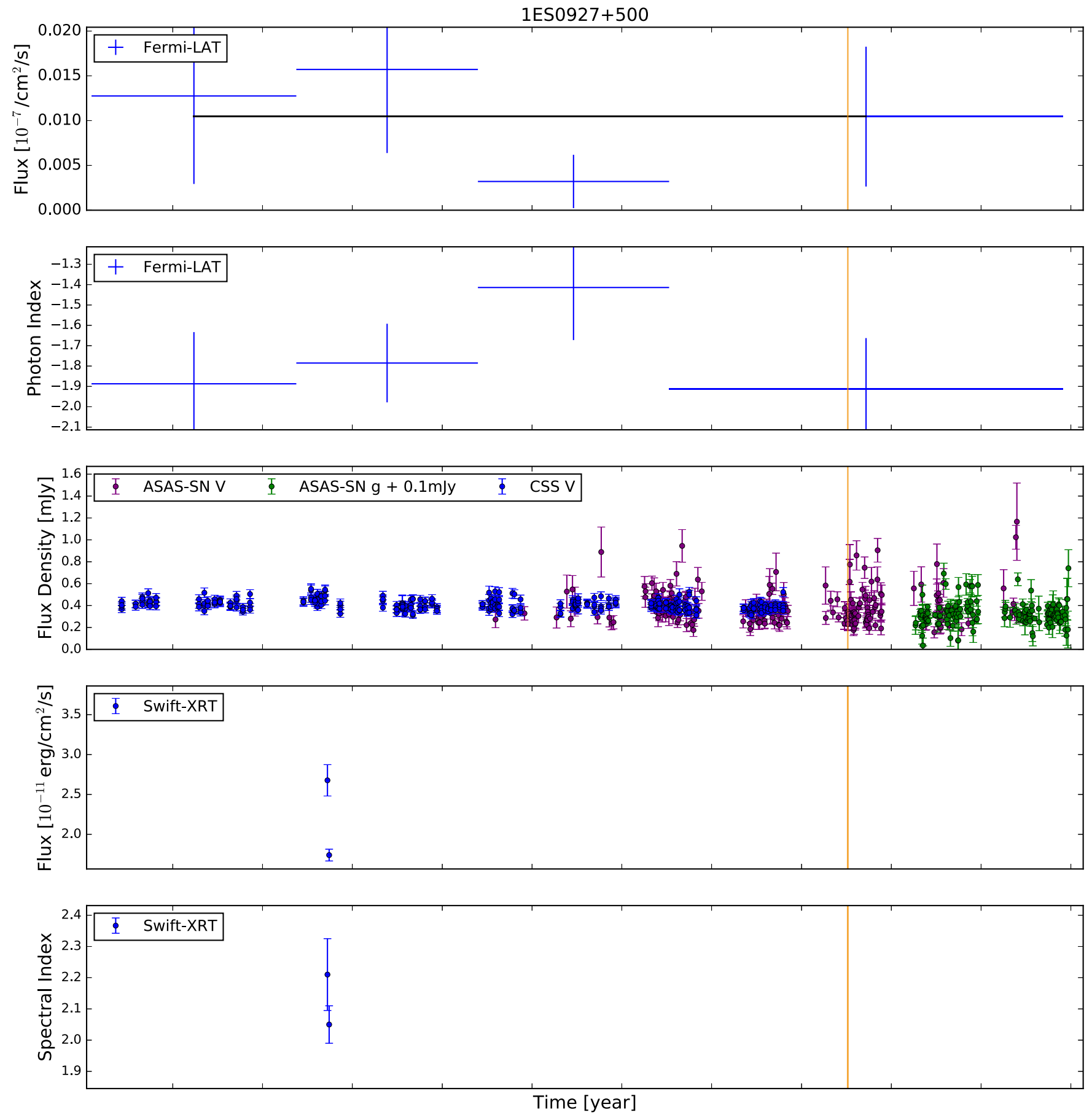

Figure A14. Multiwavelength light curve of 1ES $0927+500$. The duration of the neutrino flare is short $\left(T_{w}=1.2\right.$ days), and its arrival time is shown as an orange line.

\section{ORCID iDs}

A. Franckowiak (1) https://orcid.org/0000-0002-5605-2219

S. Garrappa (1) https://orcid.org/0000-0003-2403-4582

V. Paliya (1) https://orcid.org/0000-0001-7774-5308

B. Shappee (1) https://orcid.org/0000-0003-4631-1149

N. L. Strotjohann (ib https://orcid.org/0000-0002-4667-6730

\section{References}

Aartsen, M. G., Abbasi, R., Abdouand, Y., (IceCube Collaboration), et al. 2013, Sci, 342, 1242856

Aartsen, M. G., Ackermann, M., Adams, J., et al. 2014, PhRvL, 113, 101101 Aartsen, M. G., Ackermann, M., Adams, J., et al. 2015, ApJ, 807, 46
Aartsen, M. G., Ackermann, M., Adams, J., et al. 2018a, Sci, 361, eaat1378 Aartsen, M. G., Ackermann, M., Adams, J., (IceCube Collaboration), et al. 2018b, Sci, 361, 147

Aartsen, M. G., Ackermann, M., Adams, J., et al. 2020, PhRvL, 124, 051103 Aartsen, M. G., Abraham, K., Ackermann, M., et al. 2016, ApJ, 833, 3

Aartsen, M. G., Abraham, K., Ackermann, M., et al. 2017a, ApJ, 835, 151

Aartsen, M. G., Abraham, K., Ackermann, M., et al. 2017d, ApJ, 835, 45

Aartsen, M. G., Ackermann, M., Adams, J., et al. 2017b, JInst, 12, P03012

Aartsen, M. G., Ackermann, M., Adams, J., et al. 2017c, APh, 92, 30

Abazajian, K. N., Adelman-McCarthy, J. K., Agüeros, M. A., et al. 2009, ApJS, 182, 543

Abdo, A. A., Ackermann, M., Ajello, M., et al. 2009, ApJL, 707, L142

Abdo, A. A., Ackermann, M., Ajello, M., et al. 2010, ApJ, 710, 810

Astropy Collaboration, Price-Whelan, A. M., Sipöcz, B. M., et al. 2018, AJ, 156,123 
Astropy Collaboration, Robitaille, T. P., Tollerud, E. J., et al. 2013, A\&A, 558, A33

Abeysekara, A. U., Alfaro, R., Alvarez, C., et al. 2013, APh, 50, 26

Ackermann, M., Ajello, M., Atwood, W. B., et al. 2015, ApJ, 810, 14

Ahn, C. P., Alexandroff, R., Allende Prieto, C., et al. 2012, ApJS, 203, 21

Alard, C. 2000, A\&AS, 144, 363

Alard, C., \& Lupton, R. H. 1998, ApJ, 503, 325

Ansoldi, S., Antonelli, L. A., Arcaro, C., et al. 2018, ApJL, 863, L10

Arnaud, K. A. 1996, in ASP Conf. Ser. 101, Astronomical Data Analysis Software and Systems V, ed. G. H. Jacoby \& J. Barnes (San Francisco, CA: ASP), 17

Atoyan, A., \& Dermer, C. D. 2001, PhRvL, 87, 221102

Atwood, W. B., Abdo, A. A., Ackermann, M., et al. 2009, ApJ, 697, 1071

Aublin, J. 2019, ICRC (Madison, WI), 36, 840

Bauer, F. E., Condon, J. J., Thuan, T. X., \& Broderick, J. J. 2000, ApJS, 129,547

Breeveld, A. A., Landsman, W., Holland, S. T., et al. 2011, in AIP Conf. Ser 1358, Gamma Ray Bursts, ed. J. E. McEnery, J. L. Racusin, \& N. Gehrels (Melville, NY: AIP), 373

Britzen, S., Fendt, C., Böttcher, M., et al. 2019, A\&A, 630, A103

Cerruti, M., Zech, A., Boisson, C., et al. 2019, MNRAS, 483, L12

Cohen, D. P., Romani, R. W., Filippenko, A. V., et al. 2014, ApJ, 797, 137

Dimitrakoudis, S., Mastichiadis, A., Protheroe, R. J., \& Reimer, A. 2012, A\&A, 546, A120

Drake, A. J., Djorgovski, S. G., Mahabal, A., et al. 2009, ApJ, 696, 870

Fermi-LAT collaboration 2019, arXiv:1905.10771

Fermi-LAT Collaboration, Abdollahi, S., Ackermann, M., et al. 2018, Sci, 362, 1031

Gao, S., Fedynitch, A., Winter, W., \& Pohl, M. 2019, NatAs, 3, 88

Garrappa, S., Buson, S., Franckowiak, A., et al. 2019, ApJ, 880, 103

Ghisellini, G., \& Tavecchio, F. 2009, MNRAS, 397, 985

Ghisellini, G., Tavecchio, F., \& Chiaberge, M. 2005, A\&A, 432, 401

Ghosh, R., Dewangan, G. C., Mallick, L., \& Raychaudhuri, B. 2018, MNRAS, 479, 2464

Henden, A. A., Levine, S., Terrell, D., \& Welch, D. L. 2015, AAS Meeting, $225,336.16$

Hewett, P. C., \& Wild, V. 2010, MNRAS, 405, 2302

IceCube Collaboration 2019a, GCN, 25225, 1

IceCube Collaboration 2019b, GCN, 24392, 1

Kadler, M., Krauß, F., Mannheim, F., et al. 2016, NatPh, 12, 807

Kalberla, P. M. W., Burton, W. B., Hartmann, D., et al. 2005, A\&A, 440, 775

Keivani, A., Murase, K., Petropoulou, M., et al. 2018, ApJ, 864, 84

Kiehlmann, S., Hovatta, T., Kadler, M., Max-Moerbeck, W., \& Readhead, A. C. S. 2019, ATel, 12996, 1

Kochanek, C. S., Shappee, B. J., Stanek, K. Z., et al. 2017, PASP, 129, 104502

Krauß, F., Deoskar, K., Baxter, C., et al. 2018, A\&A, 620, A174

Krawczynski, H., Hughes, S. B., Horan, D., et al. 2004, ApJ, 601, 151

Kun, E., Biermann, P. L., \& Gergely, L. Á. 2017, MNRAS, 466, L34
Kynoch, D., Landt, H., Ward, M. J., et al. 2018, MNRAS, 475, 404

Liu, R.-Y., Wang, K., Xue, R., et al. 2019, PhRvD, 99, 063008

Lott, B., Escande, L., Larsson, S., \& Ballet, J. 2012, A\&A, 544, A6

Mannheim, K. 1993, A\&A, 269, 67

Mannheim, K. 1995, APh, 3, 295

Mannheim, K., Stanev, T., \& Biermann, P. L. 1992, A\&A, 260, L1

Mastichiadis, A. 1996, SSRv, 75, 317

Mattox, J. R., Bertsch, D. L., Chiang, J., et al. 1996, ApJ, 461, 396

Murase, K. 2017, Neutrino Astronomy: Current Status, Future Prospects (Singapore: World Scientific), 15

Murase, K., Guetta, D., \& Ahlers, M. 2016, PhRvL, 116, 071101

Oikonomou, F., Murase, K., \& Petropoulou, M. 2019, EPJ Web Conf., 210, 03006

O’Sullivan, E., \& Finley, C. 2019, ICRC (Madison, Wi), 39, 358, ICRC2019

Padovani, P., Giommi, P., Resconi, E., et al. 2018, MNRAS, 480, 192

Padovani, P., Oikonomou, F., Petropoulou, M., Giommi, P., \& Resconi, E. 2019, MNRAS, 484, L104

Paiano, S., Falomo, R., Treves, A., \& Scarpa, R. 2018, ApJL, 854, L32

Paiano, S., Padovani, P., Giommi, P., Scarpa, R., \& Treves, A. 2019, ATel, 13202, 1

Paliya, V. S., Parker, M. L., Jiang, J., et al. 2019, ApJ, 872, 169

Paliya, V. S., Sahayanathan, S., Parker, M. L., et al. 2014, ApJ, 789, 143

Petropoulou, M., Murase, K., Santander, M., et al. 2020, ApJ, 891, 115

Plavin, A. V., Kovalev, Y. Y., Kovalev, Y. A., \& Troitsky, S. V. 2020, arXiv:2001.00930

Protheroe, R. J. 1999, NuPhS, 77, 465

Reimer, A., Böttcher, M., \& Buson, S. 2019, ApJ, 881, 46

Richards, J. L., Max-Moerbeck, W., Pavlidou, V., et al. 2011, ApJS, 194, 29

Righi, C., Tavecchio, F., \& Pacciani, L. 2019, MNRAS, 484, 2067

Rodrigues, X., Gao, S., Fedynitch, A., Palladino, A., \& Winter, W. 2019, ApJL, 874, L29

Ros, E., Kadler, M., Perucho, M., et al. 2020, A\&A, 633, L1

Scargle, J. D., Norris, J. P., Jackson, B., \& Chiang, J. 2013, ApJ, 764, 167

Schlafly, E. F., \& Finkbeiner, D. P. 2011, ApJ, 737, 103

Shappee, B. J., Prieto, J. L., Grupe, D., et al. 2014, ApJ, 788, 48

Stecker, F. W., Done, C., Salamon, M. H., \& Sommers, P. 1991, PhRvL, 66,2697

Szabo, A. P., \& Protheroe, R. J. 1994, APh, 2, 375

Tavecchio, F., Ghisellini, G., \& Guetta, D. 2014, ApJL, 793, L18

Tody, D. 1986, Proc. SPIE, 627, 733

Tody, D. 1993, in ASP Conf. Ser. 52, IRAF in the Nineties, ed. R. J. Hanisch et al. (San Francisco, CA: ASP), 173

Wood, M., Caputo, R., Charles, E., et al. 2017, ICRC (Bexco, Busan, Korea), 301, 824, ICRC2017

Zhang, B. T., Petropoulou, M., Murase, K., \& Oikonomou, F. 2020, ApJ, 889,118

Zhang, H., Fang, K., Li, H., et al. 2019, ApJ, 876, 109

Zhou, H., Wang, T., Yuan, W., et al. 2007, ApJL, 658, L13 